\title{
Nanomaterials-Based siRNA Delivery: \\ Routes of Administration, Hurdles and Role of Nanocarriers
}

\author{
Nitin Gupta, Divya Bharti Rai, Ashok Kumar Jangid, Deep Pooja, \\ and Hitesh Kulhari
}

\begin{abstract}
Ribonucleic acid interference (RNAi) is a potential alternative therapeutic approach to knock down the overexpression of genes in several disorders especially cancers with underlying genetic dysfunctions. For silencing of specific genes involved in cell cycle, small/short interfering ribonucleic acids (siRNAs) are being used clinically. The siRNA-based RNAi is more efficient, specific and safe antisense technology than other RNAi approaches. The route of siRNA administration for siRNA therapy depends on the targeted site. However, certain hurdles like poor stability of siRNA, saturation, off-target effect, immunogenicity, anatomical barriers and non-targeted delivery restrict the successful siRNA therapy. Thus, advancement of an effective, secure, and long-term delivery system is prerequisite to the medical utilization of siRNA. Polycationic nanocarriers mediated targeted delivery system is an ideal system to remove these hurdles and to increase the blood retention time and rate of intracellular permeability. In this chapter, we will mainly discuss the different biocompatible, biodegradable, non-toxic (organic, inorganic and hybrid) nanocarriers that encapsulate and shield the siRNA from the different harsh environment and provides the increased systemic siRNA delivery.
\end{abstract}

Keywords Naked siRNA · Overexpression of genes $\cdot$ Hurdles/barriers · Systemic delivery $\cdot$ Cationic nanocarriers $\cdot$ Targeted delivery

N. Gupta $\cdot$ D. B. Rai $\cdot$ A. K. Jangid $\cdot$ H. Kulhari $(\bowtie)$

School of Nano Sciences, Central University of Gujarat, Gandhinagar, Gujarat, India

e-mail: hitesh.kulhari@cug.ac.in

D. Pooja

Applied Biology Division, CSIR-Indian Institute of Chemical Technology,

Hyderabad, Telangana, India 


\section{Introduction}

In recent years, one of the most important transformations in the area of biology has occurred as RNA molecular biology. In 1990, RNA interference (RNAi) phenomenon was firstly discovered in plants and Caenorhabditis elegans (nematode) in the form of single-stranded RNA (ssRNA) by Carolyn Napoli and Richard Jorgensen. Later, in 1998, Andrew Fire and Craig Mello found RNAi as a double-stranded RNA (dsRNA) in mammalian cells; pursue the development of an innovative therapeutic tool in the biomedical field. Andrew Fire and Craig Mello demonstrated that dsRNA is more effective for down-regulation of gene expression than ssRNA (Xu and Wang 2015; Fire et al. 1998). The interference mechanism of siRNA is involved to control many cellular phenomena including an intranuclear arrangement of heterochromatin and development, multiplication, differentiation and proliferation of cells (Berezikov 2011). The core of mechanism is RNA molecules; microRNA (miRNA), siRNA, and germline-directed Piwi-interacting RNA (piRNA) which actually directs degradation of the protein-encoding messenger RNA (mRNA) through a complex of certain enzymes. While piRNA and microRNA intrinsically generate from the genome, the siRNA perform as an exogenously designed fragment for interference therapy (Wilson and Doudna 2013) Thus, post-transcriptional gene silencing regulates the outcome of the targeted gene by repressing translation.

siRNA has several merits over conventional therapeutic agents like synthetic drugs or bioflavonoids. The key advantages are remarkable target-specific gene knockdown/silencing property, lesser side effects, potent gene regulation, thermodynamic stability, ease of synthesis with low production costs than protein or antibodies and there is no need of cellular expression system and complex protein purification (Liu et al. 2004; Chougule 2012). One of the major advantages of RNAi is the capability to access "non-druggable" targets; for instance, certain targeted proteins which become inaccessible to drug due to alteration in enzymatic function or conformation. For such cases, synthetic RNA can be sequenced to target almost any desired gene of human genome (Aagaard and Rossi 2007). This advancement is associated with the emergence of short noncoding RNA stretch known as siRNA. Because of its unique property to regulate the targeted genes expression (invivo and in-vitro both), currently siRNA has obtained more attention as a potent therapeutic agent in several biological and genetic abnormalities such as neurodegenerative diseases, Huntington's disease, hematological diseases, inherited genetic disorders, dominant genetic disorders, cardiovascular disorders, various cancers, viral infections, autoimmune diseases, ocular diseases and many other illnesses caused by action from one or several genes, which can be regulated via RNAi phenomenon (Gavrilov and Saltzman 2012; Dorn et al. 2004; Jagannath and Wood 2007). The genetic expression may be altered at various genetic levels, involving RNA processing, RNA stability, chromatin structure, chromosome segregation transcription, and translation (Carthew and Sontheimer 2009) 
siRNA is a better therapeutic agent than conventionally used therapeutic agents. For the effective therapeutic therapy, the first and most essential step is to ensure the delivery of siRNA to the targeted/site-specific tissue and cells from the administrated site. Although, the systemic circulation of naked RNA molecule is challenged with several hurdles/problems such as fast enzymatic degradation in the physiological and biological media, entrapment by phagocytes, extravasation from blood to tumor tissues, recognition by the immune system, renal clearance, poor tissue penetration, incompetent endocytosis, immunostimulation, and off-target effects. These several drawbacks prevent the delivery of siRNA to the active site (Lee et al. 2016; Gewirtz 2007). So, viral and non-viral carriers developed as carrier systems. Initially, the viral vectors were used as delivery platforms where the viruses deliver the therapeutic siRNA into the targeted cells through the mechanism of transduction. They are suitable for both transient and long-term gene silencing, depending on the type of carrier viruses. Moreover, viral vectors have the ability to enter the nucleus of the cells (Qiu et al. 2016a). However, these conventional vectors have a risk of causing immunogenicity, toxicity, and insertional mutagenesis. The smaller size of viral particles with small size also limits the size of a therapeutic gene that can be inserted and carried. Some of the carriers and conjugated with various molecules are used to deliver siRNA such as aptamer oligonucleotide, sterol or antibody conjugates with siRNA. But these conjugations and carriers are not enough for the systemic delivery of siRNA.

Therefore, for overcoming the associated hurdles during systemic delivery and to successfully deliver siRNA-based drugs in-vivo with easy and improved extensive practical applications, various carriers or vectors are required that can convey the siRNA to the desired site of activity. Non-viral based biocompatible, biodegradable, non-toxic, nano-sized carrier systems extensively employ nanomaterials (NMs) as compact carriers for in-vivo delivery of siRNA. NCs have the diameter/size $<100 \mathrm{~nm}$. Due to small size, NCs have some advanced and unique physiochemical properties than micro or macro carriers such as they possess a greater ratio of surface area to volume with the increased number of surface-active molecules. These nanocarriers (NCs) form complexes with the siRNA or encapsulate it to develop an intact and inert molecule or particle which that is capable of transfecting the targeted cells and inducing the genetic interference (Dana et al. 2017). Davis et al. (2010) showed the site-specific transport of siRNA using NCs in humans via systemic injection in earlier work, which developed as a foundation for later research related to the medical application of siRNA (Davis et al. 2010). After this, several researchers and scientists have developed/synthesized several NCs such as cationic polymeric nanoparticles (polyplex), liposomal nanocarriers (lipoplex), carbon nanomaterials, lipid-based nanomicelles etc. These NCs protect the siRNA from rapid enzymatic degradation in biological and physiological media, reduce immunogenicity, increase blood retention time and cellular uptake, and increase the targeted delivery. These advantages are responsible for improving the therapeutic effects of siRNA during systemic circulation. 
This chapter presents the basic mechanism of siRNA interference, its advantages, various routes of administration, hurdles/challenges during systemic delivery naked siRNA, and overcoming of these hurdles using various nanocarriers.

\subsection{Mechanism of siRNA Interference}

siRNA interference is an intrinsic process of post-transcriptional gene regulation where a non-coding stretch of RNA sequence down-regulate the activity of several coding genes and silence the gene expression (Draz et al. 2014). Mostly a short dsRNA fragment known as siRNA mediates homology-based regulation of gene expression in eukaryotic cells (Fig. 1) (Almeida and Allshire 2005). This process can be compared to another approach based on miRNA knockdown and replacement. For inhibition, a synthetic ssRNA known as antagomirs/anti-miRs employed as the antagonist of intrinsic miRNA through an antisense mechanism. The replacement process involves synthetic miRNAs which are introduced in-vivo to mimic the function of the endogenous miRNAs. This RNA mimicking leads to gene silencing and degradation/inhibition of mRNA consequently. This chapter elaborates the clinical application of RNA interference in medical treatment and exclusively focuses on the siRNA therapy (Aagaard and Rossi 2007).

The first step of RNAi is the initiation phase which involves the production of effectors molecules. There are two specific nucleotides at the $3^{\prime}$-overhang of dou-

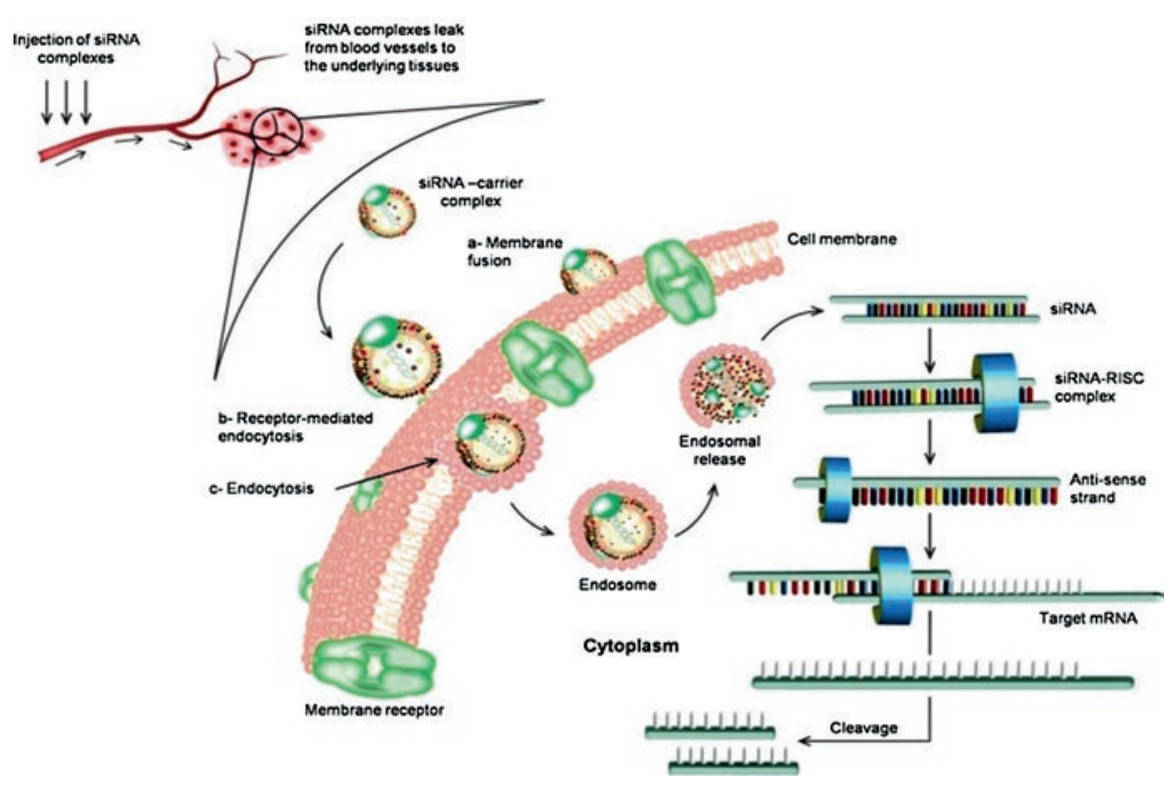

Fig. 1 Mechanism of siRNA interference in-vivo. (Draz et al. 2014) 
ble-stranded RNA molecules approximately 21-22 base pair long which is targeted by an enzyme complex known as Dicer, in homology-dependent mechanism to cleave recognized mRNA. The RNase III-like enzyme termed as Dicer, degrades the longer dsRNA, generating short interfering RNAs in the homology-dependent mechanism (Gavrilov and Saltzman 2012). This processing of dsRNA into siRNA constructs by endonuclease Dicer occurs in the cell cytoplasm.

After the formation of effector molecules, the execution phase begins when a multiprotein complex bound the effector molecules to stimulate gene silencing. siRNAs are bound by a multi-subunit assembly of certain proteins forming RNAinduced silencing complex (RISC) (Hammond et al. 2000). The RISC complex includes three proteins, namely Dicer, TRBP, and Argonaute 2, each with a distinct role; (1) Dicer joins siRNAs to the RISC (2) TAR-RNA binding protein (TRBP) assists the siRNA-RISC bounding (3) Ago-2 is the slicing protein of RISC complex that acts at specific cleavage site between bases 10 and 11 from the $5^{\prime}$-terminal of the antisense siRNA strand, in the target mRNA molecules (Aagaard and Rossi 2007). The mRNA strands which are almost complementary of guide RNA strand recognized and sliced by Ago-2 protein. In the second instance, this complementary siRNA-mRNA pair sometimes resembles miRNA bounded to target sites. miRNAs are processed within the nucleus by Drosha-DGCR8 complex, thereby repressing translation or degrading the transcript.

The Argonaute (Ago) proteins are the sole member of RISC and the catalytic domain of Ago-2 enables the cleavage of targeted mRNA region (Meister et al. 2004). The siRNAs embedded within RISC are duplex, Ago-2 proteins detach and remove one of the strands from RISC bounded double-stranded siRNA referred to as the passenger strand. The other single strand, known as guide RNA, remains embedded in the RISC complex (Fig. 2). The functionally active complex with guide RNA recognizes the target due to specific intermolecular base pair interaction (Tang 2005). Ago-2 protein is composed of three functional subunits; namely PIWI, PAZ, and MID. Among these, the PIWI domain possesses the structural folding similar to RNase $\mathrm{H}$ and provides thermodynamic enthalpy to the RISC complex for

Fig. 2 Formation of the RISC complex. (Gavrilov and Saltzman 2012)
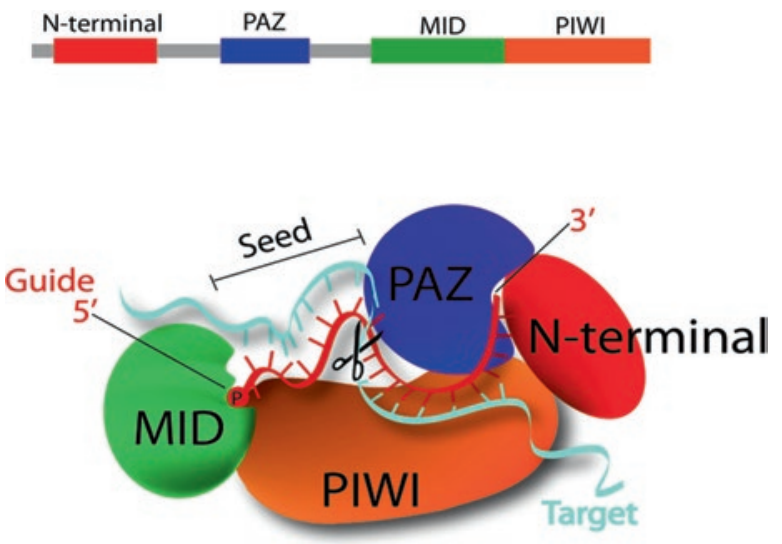
cleavage (Song et al. 2004). The PAZ subunit of the Ago-2 protein binds two terminal 3'-nucleotide specifically for connecting it to the RISC complex. The staggered ends of the siRNA consisting of the specific nucleotide lie in the core domain in a hydrophobic cavity composed of various aromatic residues where the base of the terminal nucleotide is stacked (Song et al. 2003). The 5'-phosphate group of siRNA is bounded to the carboxyl-end of the protein in the middle of the MID and PIWI domains through a magnesium ion (Ma et al. 2005). It is observed that the Argonaute family also contributes while guide-strand selection. Argonaute conserves the strand which has less thermodynamically stable $5^{\prime}$-terminal as the guide strand and cleaves another one to release the passenger strand (Schwarz et al. 2003).Certain basic principles such as thermodynamic stability of the siRNA end regulate the selection of the strand which remains anchored to the RISC complex (Schwarz et al. 2003). The strand with less stability at the terminals usually converts to form mRNA molecules which can be recognized and later on degraded by Ago- 2 . The strand complementary to the targeted mRNA act as guide RNA.

\section{Advantages of siRNA}

RNAi has several extra benefits and advantages compared to others therapeutic agents (already discussed in Sect. 1). However here, we are discussing some of the significant advantages of siRNA in details including its therapeutic efficacy/potency for suppressing of gene expression, self-targeted/selective delivery on infected cells, and safety.

\subsection{Potency}

Contrary to other bioactive molecules applied so far including antisense DNA oligonucleotides and RNA-enzymes, RNAi has a greater potential in antisense technology (Bertrand et al. 2002). The high potential of RNAi approach observed because of the fact that effector molecules may function at a lower concentration than in the case of ribozymes or antisense oligos. This is a major advantage in a clinical scenario. The underlying factor for the above success of RNAi over other antisense-based practices for treatment purpose is that it autonomously target the complementary transcripts by using the host cellular machinery, often leading to suppression of gene expression up to much extend. Of note, small antisense ssRNAs may also bind complementarily into RISC and conduct target mRNA (Martinez et al. 2002).

The efficiency of siRNA interference is decided by many parameters such as structural features (Patzel et al. 2005), target mRNA accessibility (Heale et al. 2005), position-specific determinants (Amarzguioui and Prydz 2004), and differential end stability (Schwarz et al. 2003). The most important parameter among is the 
thermodynamic stability of terminal points which depends on asymmetry and holds an important function in the selection of mRNA. This efficiency is usually measured by half of the maximum inhibition value known as $\mathrm{IC}_{50}$ values.

\subsection{Selectivity}

The high degree of specificity of siRNA-based approach is a major quality which enables the recognition of targeted and non-targeted sequences. The basis of the selectivity is the complementarity of mRNA bases, also referred to as Watson-Crick base pairing interactions. So, the antisense strand is designed based on targeted mRNA sequences and hypothetically. It is possible to treat all kind of genetic disorder through RNAi method once we know the nucleotide sequence of the associated genes. This unlimited capability of antisense-mediated suppression has supported systems for mutating nearly all the known genes of protein production comprised in the human genome at an extensive range. The high specificity may even permit distinguishing between abnormal alleles of a gene which arises dysfunction from the native allele which may differ in just a single or few nucleotide bases. This is obviously helpful for focusing on predominant mutants, as an example in the case of a few oncogenes (Aagaard and Rossi 2007).

Furthermore, certain malignancies such as cancer can result in different etiology such as abnormal chromosomal number, multiple gene mutations, or epigenetic changes (Clark 2007). So, this disease may be significantly different in the case of each patient and requires personalized treatment. Moreover, cases not responding to chemotherapy method due to resistance also may get the advantage of siRNA therapy (Gottesman 2002).

\subsection{Safe and Cheap Alternative}

The RNAi effector molecule executes the cleaving of mRNA at the later stage of translational gene expression, unlike other antisense molecules. So there is no chance of interaction of siRNA with DNA and thereby deleterious mutation and pre-birth abnormalities due to gene therapy (Xu and Wang 2015). As well as the process is used to synthesized siRNA is easy and cheaper. For the synthesis of RNAi, no any costly instruments and hazardous chemical are used. 


\section{Routes of Administration of siRNA}

There have been various clinical trials reported in RNAi therapy by local and systemic administration of siRNA to various organs such as kidney, eye, liver, and skin which is dictated by the types of cells and tissues at the targeted site. For localized delivery, siRNA can be administered specifically to the skin surface, eye, lungs etc. On the other hand, oral, intravenous (IV) or intraperitoneal (IP) routes of systemic delivery are suitable for those genetic dysfunctions which are not confined to a particular site or not easily amenable to other clinical therapies. But the systemic siRNA administration has many hurdles such as less specificity and bioavailability to the targeted sites, rapid clearance or systemic toxicity (Durcan et al. 2008).

\subsection{Localized Delivery}

Administration at the local sites has turned into better and compelling option, permitting the utilization of lower dosages and diminishing the undesirable effects. siRNA specifically administered through different routes such as topically, gastrointestinal tract (GIT), respiratory tract, nervous system, ocular etc. The proficient administration of naked siRNA has been done through the topical application at the anterior chamber or by injecting within the vitreous at the posterior chamber. Particularly as a localized manner, delivery of siRNA to through the topical and ocular surface is easy compared to other organs, tissues, and cells. Continuous progress has been made towards its use as a therapeutic procedure for eye diseases. Currently, certain siRNA-based drugs for topical and ocular defects have passed the preclinical phase and various clinical trials studies are going on (Nguyen et al. 2012a, b; Pañeda 2013).

\subsubsection{Topical Administration}

The skin is the outermost covering of the body with the largest surface area, hence prone to numerous skin diseases including some genetic defects as well such as dermatitis, rheumatoid arthritis, psoriasis, pachyonychia congenital, alopecia areata, melanoma, wounds etc. Topical application is most beneficial for the treatment of these disorders due to easy accessibility at the affected site, decreased systemic side effects, elimination of the first-pass metabolism, thereby decreasing the therapeutic dose. Being a non-invasive and easy-to-administer method, the topical application also enhances patient's compliance to treatment (Prausnitz et al. 2004).

siRNA module has been tried for the treatment of psoriasis which is one of the most common skin disorders and associated with overgrowth in keratinocytes due to declination in apoptosis that makes a plaque in addition to the inflammatory component of the disease. Conventional treatment of psoriasis topically applies cortico- 
steroids, which leads to weight gain, itching dryness, stinging, fluid retention, irritation, hypopigmentation, atrophy, hypertension, and other side effects. It is highly desirable to find drug molecules with fewer side effects. Therefore, targeting the genetic cause i.e. upregulation of the pro-inflammatory cytokines is a more suitable way to treat psoriasis. The overproduction of cytokine can be regulated by suppressing tumor necrosis factor alpha protein. Animal studies show that silencing of gene encoding tumor necrosis factor-alpha resulted in the improvement in the psoriatic lesion.

Another successful example of the clinical utility of siRNA over drugs is observed in the case of allergic contact dermatitis (ACD). Only a short-term treatment with topical corticosteroids and calcineurin inhibitors were available earlier. However, the development of siRNA molecules in recent years has enabled more specific targeting of genetic factors. ACD is genetically characterized by the elevated expression of CD86 in the affected skin area. The reduction of CD86 expression by RNAi silencing decrease the inflammatory response of the immune system and hypersensitivity (Aldawsari et al. 2015).

Despite the above advantages, there are several anatomical barriers due to composition and cellular distribution of the superficial surface of the skin. The outer stratum corneum is impermeable to the therapeutic RNA and acts as a barrier in topical administration (Madison 2003). The issue of low penetration overcome by using carrier systems for delivering which can encapsulate therapeutic siRNA molecule and penetrate through stratum corneum to reach the target site within the dermal layers First, the carrier system should be able to encapsulate or form a complex with siRNA; second, the carrier system must possess the ability to cross the stratum corneum to reach the target cells in the skin. More importantly, the carrier system should be able to bypass endolysosomal degradation before reaching the nucleus to obtain the clinical impact of RNAi after topical application (Aldawsari et al. 2015).

\subsubsection{Ocular Administration}

Ophthalmology is the earliest field where the efficiency of RNAi-based therapeutics was studied through clinical trials. Gene therapy has proven to be most successful in the case of ocular complexities due to certain unique properties. It is an immunogenically-privileged and easily assessable site. Moreover, it is comparatively a distinct part from the rest of the body (Conley and Naash 2010). Hence, the low therapeutic dose is required and the probability of systemic toxicity also confines within the local area by subsequently enabling targeted delivery. Thus, the RNAi method can be employed as a proficient treatment for different visual impairments such as irreversible blindness (Conley and Naash 2010). siRNA-based therapy has introduced advancement to resolve visual impairments results due to age-dependent macular degeneration, glaucoma and photoreceptor degeneration been utilized to distinguish qualities that advance harm in the eye and could be the premise of new 
medications for some, sicknesses, including glaucoma, age-related macular degeneration, and photoreceptor degeneration (Campochiaro 2006).

For example, major ophthalmic disorders in humans such as anterior ischemic optic neuropathy and glaucoma-related blindness are the consequences of Retinal ganglion cell (RGC) destruction is efficiently treated by intravitreal administration of siRNA. The studies on rat models suggest that loss of RCG is due to cleavage by a caspase- 2 nuclease, which is unregulated in case of optic nerve damage. Intravitreal delivery of a chemically-modified synthetic siRNA for inhibiting the expression caspase- 2 expression enhanced RGC survival remarkably. The persistence of exogenously delivered siRNA has a longer span of up to a month and a safer RNA interference without prompting interferon-mediated inflammation in the retinal region which is immunogenically-privileged, contrary to the conventional ophthalmic drug formulations which sometimes cause ocular irritation. The trials of RNAi resulted in suppression of caspase-2 enzyme and thus siRNA are clinically proven as the latent neuroprotective component for intervention in ocular diseases concerning to RGC loss (Ahmed et al. 2011).

Despite being a suitable site for targeted siRNA release, few problems have been observed in ocular administration. The first physical barrier is protective viscous fluid secrete by the lacrimal gland and known as tear film. It continually washes of the applied formulation before complete absorption. The second hindrance is produced by conjunctival and corneal epithelial cells which have low penetrability (de la Fuente et al. 2010). Similar hurdle is produced by a blood-retinal barrier (Bodor and Buchwald 2005). Because of the mentioned physiological constraints, ocular administration of interfering RNA suffers from low bioavailability. Therefore, delivery of RNA via ocular site depends on the efficient delivery platform.

\subsubsection{Pulmonary Administration}

Lungs are the most vital organ of the respiratory system and attribute to special morphological features such as large surface area and vascularized structure. These features enable the site-specific administration to lungs through aerosol formulation or intranasal instillation and provide novel methods for curing respiratory related disease (Thomas et al. 2007). Also, siRNA is prevented from degradation by nuclease during pulmonary administration through the respiratory tract. However, there are certain physical hurdles, for example, beating moment of cilia and flow of mucus for clearance of respiratory route epithelial cells and the negatively charged cell membrane surface (Gutbier et al. 2010). These hindrances influence the proficiency of in-vivo delivery of siRNA (Griesenbach et al. 2006). Numerous investigations have examined in spite of the above troubles, the scope of localized administration for site-specific application of siRNA to the organs of the respiratory system. Such investigations discover new medical strategies for major respiratoryassociated disorders, for example, influenza, respiratory syncytial infection, cancer and severe acute respiratory syndrome (SARS), atypical mycobacterial infections, lung cancer, respiratory alphaherpesvirus infection, pulmonary fibrosis, asthma, 
chronic obstructive pulmonary disorder (COPD), emphysema, respiratory syncytial virus (RSV), parainfluenza virus (PIV), rhinoviruses coronavirus (SCV), ischemic reperfusion injury and other endogenously accessible disease in the lungs by confined delivery at peculiar site. Pulmonary administration can be done by inhalation, intranasal route, and intra-tracheal route.

Localized delivery of siRNA in lung disorders provided an alternative when the systemic administration does not efficiently suppress the targeted proteins. Most common example of commercial siRNA formulation with pulmonary administration method is ALN-RSV01, used in case of upper respiratory tract infection which occurs due to the growth of RSV. This formulation acts on the $\mathrm{N}$ gene and inhibit the nucleocapsid synthesis in RSV, thereby control the replication of RSV in nasal passages and trachea.

Cystic fibrosis is another genetic disorder which involves the chronic inflammation mediated through $\mathrm{IkB} /$ protein complex that is involved in transcriptional regulation of DNA, cytokinesis and cellular development. This protein is produced by cystic fibrosis trans membrane conductance regulator (CFTR) gene and epithelial $\mathrm{Na}^{+}$transporter (ENaC) gene which founds to be mutated in the case of cystic fibrosis. Pulmonary siRNA delivery decreases the expression of epithelial $\mathrm{Na}^{+}$channel $(\mathrm{ENaC})$ gene or down-regulate its activity to normalize the $\mathrm{Na}^{+}-\mathrm{Cl}^{-}$transport across the cell membrane and prevent the excess mucus release, luminal hydration, pulmonary infection and epithelial cell destruction (Khatri et al. 2012).

\subsubsection{Gastrointestinal Administration}

siRNA particularly could be administered within the gastrointestinal system by means of endoscopic infusion for the treatment of infections. The surface of the GI tract is covered by a layer of fluid called mucus. This viscous fluid stimulates the release of RNAi formulation straightforwardly to its site of activity, thereby eliminating any undesirable effects and systemic toxicity to adjacent tissues and organs (Pellish et al. 2008). Moreover to the application of siRNA for treatment GI-associated diseases, this strategy also has been utilized for illustrating the course of different gastrointestinal infections and the determination of possible targets for RNA interference, for example, tumour necrosis factor alpha (TNF- $\alpha$ ) in the case of ulcerative colitis, Crohn's disorder and inflammatory bowel diseases (IBD) (Zhang et al. 2006). IBD earlier treated with infliximab, a chimeric IgG-1 monoclonal antibody against (TNF- $\alpha$ ). But these antibodies can trigger anti-antibodies production and elicit immune hypersensitivity. On contrary, localized silencing of TNF- $\alpha$ through siRNA molecules may not trigger any immune response.

Traditional treatment of oesophageal cancer involves aggressive surgical and adjuvant chemoradiation therapy. One common etiology observed in various types of oesophageal malignancies is hyperactivity of $\mathrm{Bcl}-\mathrm{X}_{\mathrm{L}}$, the gene responsible for inhibiting apoptosis. Therefore, by interfering with the $\mathrm{Bcl}-\mathrm{X}_{\mathrm{L}}$ expression via siRNA-based approach, the oesophageal squamous cell cancer line was destructed in the apoptotic programme. 
Similar to oesophageal malignancies, pancreatic cancer has a delayed diagnosis and chemotherapy practices. The common reason observed for adenocarcinoma is an elevated level of serine-arginine protein kinases (SRPK) which led to erroneous splicing of pre-mRNA is the causative in certain malignant cancer including pancreatic cancer. Because serine-arginine protein kinases (SRPK) are the class of proteins with a regulatory role in pre-mRNA splicing, and elevation in the expression of SRPK1, trials were done by Hayes et al. utilized SRPK1-specific siRNA to block SRPK1 activity. Consequently, growth in pancreatic and colonic cancer cell lines declined and apoptotic death was much higher as compared to common anticancer drugs such as gemcitabine and cisplatin (Pellish et al. 2008).

\subsubsection{Central Nervous System Administration}

The brain is the most complex organ of the body, not only regarding anatomy and function but also in terms of medical therapies. Due to its complexity contributed by various types of cells and vivid functions, the conventional treatment methods do not prove to be as effective in the case of neuron-associated problems. In this specific situation, therapeutics based on RNAi could identify the genetic basis and comprehend the course of neural disorders as a part of prognosis. Later, it could evaluate the remedial capability of targeted RNAi therapy for silencing genes involved in neurological disorders. For example, PTEN-induced putative kinase 1 (PINK1) is targeted for treatment of Parkinson's disease (Deng et al. 2005); Huntington gene for Huntington's disease (Harper et al. 2005); purinoceptor 3 (P2X3) for neuropathic pain (Dorn et al. 2004) and EGFR for intracranial tumors (Boado 2005). Certain neurodegenerative diseases such as Alzheimer's diseaseBACE1 (Singer et al. 2005); amyotrophic lateral sclerosis-SOD1 (Ralph et al. 2005) and other dysfunctions mediated by hypoxic/ischemic events could also be treated through siRNA-mediated silencing. Contrary to the potential specificity of the RNAi method, the efficiency is compromised due to the highly impermeable bloodbrain barrier that confines the availability of RNAi-based formulations across the brain from peripheral blood vessels (Cazzin and Ring 2010).

Alzheimer's disease (AD) is a protein misfolding disorder caused by defective extracellular amyloid $\beta$-peptide $(\mathrm{A} \beta)$ disease and leads to progressive cognitive decline and memory loss. The researcher has found that $\gamma$-secretase which cleaves $\gamma$-site of the amyloid precursor protein (APP) is a key component that generates A $\beta$ from through sequential proteolytic cleavage events. BACE1 is the gene expressing for $\gamma$-secretase, hence the therapeutic siRNA molecule targeting BACE1 considered as a potential gene therapy of AD. Similarly, a targeted decrease in APP expression through the same mechanism also serves the purpose.

Huntington's disease (HD) is another neurodegenerative disease benefited by this new siRNA module. The disease is associated with the upregulation of Huntingtin $(\mathrm{Htt})$ gene, which adds extra polyglutamine stretch in exon1 region of the HTT protein product. This mutant protein type misfolds and forms inclusion bodies. The striated neurons are the most degenerated cell types, hence CNS is 
severely affected in the case of HD. Silencing mutant Htt mRNA through RNAi the approach has been seen for clinical utilization (Gherardini et al. 2014).

\subsubsection{Vaginal Administration}

The vaginal route is the primary site of invasion for some microbial agents causing infections especially sexually transmitted diseases, cancer, and inflammatory problems. In such instance, the topical administration of RNA-based product is the most ideal approach to anticipate sexually transmitted diseases. This type of application provides benefits of lower therapeutic dose and maximum bioavailability at the site infection (Rossi 2009). Therefore, the studies are on-going in context of targetedsiRNA therapy using identified gene targets such as CCR5 for human immunodeficiency infection (HIV); E6, E7, and Grb10 for cervical tumor and UL-29ANDUL-27 for HSV-2 infection. However, the most usual drawback of vaginal application is patient compliance.

HIV is one of the initial viral diseases, where targeting by RNAi, was effective in treating the viral infection as well apart from genetic disorders. The siRNA molecule developed to target different viral genes including gag, tat, nef, vif, rev and env involved in the viral cycle of HIV. The RNA may also act on host cellular cofactors such as CD4, CXR4, NFkB, and CCR5 as well as reverse transcriptase and control HIV replication effectively.

The vaginal administration has been more successful in case of cervical cancer result due to overexpression of HPV oncogene and triggers unregulated proliferation of cells. Therefore, inhibiting the activity of HPV gene via siRNA is very effective in slowing the proliferation of cancerous cervical cells. The siRNA therapy can specifically target human HPV 16/18 E6/E7 genes which have a significant effect in stopping the multiplication of cervical tumors (Wu et al. 2011).

\subsection{Systemic Delivery}

Comparatively, systemic delivery is more suitable in the clinical situation of bloodborne disorders and malignancies. Thus, the recent researches are more focusing on the alternatives to bring out the systemic delivery of siRNA as a more efficient and safe procedure of gene silencing. The chapter further discusses the role of NCs to overcome the pitfalls of systemic siRNA administration. Various nanoparticles can be used as non-viral vectors to facilitate delivery rather than naked siRNA molecule. 


\subsubsection{Oral Administration}

The oral route is the most ideal mode of administration due to patient compliance. Moreover, other benefits such as cost-effectiveness and facile administration by oral route attract attention for drug delivery and are also preferred for local administration of intestinal therapeutics. But the acidic environment of stomach readily degrades the naked oligonucleotide. Therefore, the stable administration via the oral route achieved by nano carrier-mediated delivery (Akhtar 2009).

\subsubsection{Intravenous Administration}

Although IV administration is a successful mode of administration for many drug formulations, it has been observed that the delivery of siRNA by this method is hampered by the very short lifespan kidney filtration, serum protein aggregation and enzymatic degradation of therapeutic RNA after injecting. It has been exhibited in mice, rats, human and other primates that bare siRNAs are rapidly cleared from the body circulation after IV infusion with the half-life as short as a few minutes or approximately $0.5 \mathrm{~h}$ also, a large accumulation of therapeutic oligonucleotide occurs in the kidney after IV administration (Huang et al. 2016).

\subsubsection{Intraperitoneal Administration}

In addition to less stability of therapeutic RNA administered through IV route, other common after-effects of repeated IV administration are phlebitis or loss of veins and risk of embolism. In this regard, the IP delivery provides a better option, especially in the case of cancers of ovaries, digestive system, and peritoneum. The IP administration moreover found to have enhanced the in-vivo stability of siRNA and enables administration of a large volume of formulations at low concentrations so as to avoid particle aggregation (Singhania et al. 2011).

Many malignant tumors which arise from the stimulation of nuclear factor-kappa $\mathrm{B}$ (NF-kB), for instance, gastric tumor shows poor prognosis and resistance to chemotherapy alone. Thus, NF-kB is a common target for RNA silencing. A study demonstrates efficient intraperitoneal administration of NF-kB p65 siRNA along with a common chemotherapeutic agent (paclitaxel) for treating peritoneal prevailing of gastric tumor. Lower expression of NF-kB p65 expression was observed in western blot analysis and apoptotic destruction of cells transfected with NF-kB p65 siRNA as compared to treatment with paclitaxel alone (Inoue et al. 2008). 


\section{Major Hurdles to the Therapeutic Delivery of siRNA}

The efficacy of RNA interference may differ for various siRNAs oligonucleotides targeting same mRNA sequence but at distinct nucleotide. Full efficiency of siRNA is not obtained as only some of the delivered siRNAs reach the action site without distortion in mammalian cells and successfully perform cleaving action (Naito and Ui-Tei 2013). Even though, approximately 58-78\% of arbitrary siRNAs with nonspecific sequences, mediate mRNA silencing with an efficacy more than half of the proficiency $(>50 \%)$. Out of this, most of the (90-95\%) proficiency is accounted by just 11-18\% of the non-specific siRNA oligonucleotides (Chalk et al. 2004).

The decreased efficacy of siRNA may have many causes including instability, unspecific targeting to diseased-cells, and competition with endogenous RNAs, saturation of RNAi/miRNA complex, immune response activation and off-target impact. The problems become more prominent in applying the systemic mode of administration for delivering siRNA which imposes some more disadvantages compared to localized administration. The main problem is to conserve the activity of siRNA during circulation in the systemic blood vessels. The systemic circulation of siRNA is further hindered due to many anatomical and physiological protective barriers present in the human body such as renal filtration, vascular endothelium, extracellular matrix, serum protein aggregation, cellular internalization, phagocytes uptake, endosomal degradation, and RISC loading. siRNA need to overcome these barriers before it reaches its site of action, to exhibit clinically significant impact with considerable proficiency.

\subsection{Transient Effect}

RNAi mediated gene silencing can be stimulated by either direct or indirect mechanism. The first involves an RNA-mediated process where an exogenous synthetic siRNAs make a complex with a 21 - base pair long duplex. This effector molecule is then targeted towards the specific cell. Meanwhile, the second approach involves an endogenous miRNA-based strategy where the longer RNA hairpin transcripts are processed intracellularly to produce the effector siRNA. While the direct method is more straightforward and efficient for gene cleavage, the impact of externally introduced, synthetically directly generated siRNA is short term. Thus, the synthetic siRNA requires to be administered repeatedly with each dose being expensive and time-consuming. The miRNA-based RNAi drugs, on the contrary, shows high stability and long-time effect. Hence a single treatment is not enough for effective gene therapy (Aagaard and Rossi 2007). 


\subsection{Stability}

Another great pitfall of siRNA is related to the in-vivo stability. The exposed siRNA are liable to hydrolysis in the extracellular compartment due to the action of various enzymes present in serum and target tissues. The half-life of naked siRNAs in serum as reported so far is several minutes up to an hour (Rettig and Behlke 2012). The siRNA molecule structurally contains a ribose sugar backbone. On contrary to a hydrogen atom as in DNA, it is the hydroxyl group at $2^{\prime}$ position of the pentose ring which is targeted by serum nucleases (Czech et al. 2011). These nucleases break the phosphodiester backbone of RNA and degrade this nucleic acid. Thus, it is a challenge to deliver intact siRNA with conserved therapeutic activity through the circulation. Meanwhile, siRNAs must not only survive in the serum but should also reach their target cells in the specific tissues for internalization to occur appropriately and to achieve required RNAi effect (Guo et al. 2010a).

\subsection{Physiological Barriers}

As the siRNA administered and introduced into the systemic circulation, its route towards the site of action is obscured by many structural and anatomical defensive barriers (Fig. 3). The step of the clearance through the kidney and reticuloendothelial system (RES) of other organs such as lung, liver, bone marrow and spleen is the primary obstruction. It entraps all the particles with a diameter larger than $100 \mathrm{~nm}$. Thus, the ideal RNA molecules to bypass renal and RES clearance should have a diameter ranging from 20 to $100 \mathrm{~nm}$ approximately. Additionally, therapeutic material within this size range shows an enhanced uptake at tumor sites passively through leaky capillaries of a pore size of 100-800 nm (Song et al. 2005).

After passing through the RES, as the siRNA enters the targeted tissue, it is hindered by local barriers present in the extracellular matrix and the layer of endothelial cells. Typically, the endothelial lining of tissues have a pore size from 4.5 to $25 \mathrm{~nm}$, hence the smallest pore can allow particles of 4-5 nm only (Rettig and Behlke 2012). In such circumstances, only naked siRNA molecules or the one conjugated with small molecular ligands easily penetrate through the endothelial cells. Also, the naked or complexed siRNA must squeeze out of the dense extracellular space to reach the surface of the target cell.

\subsection{Cellular Uptake and Endosomal Engulfing}

Even after bypassing the nuclease activity in serum and extracellular matrix, the RNAi pathway still hindered by various obstacles. After reaching proximity to the target cells, uptake of siRNA by the target cells is also crucial, prior the therapeutic 


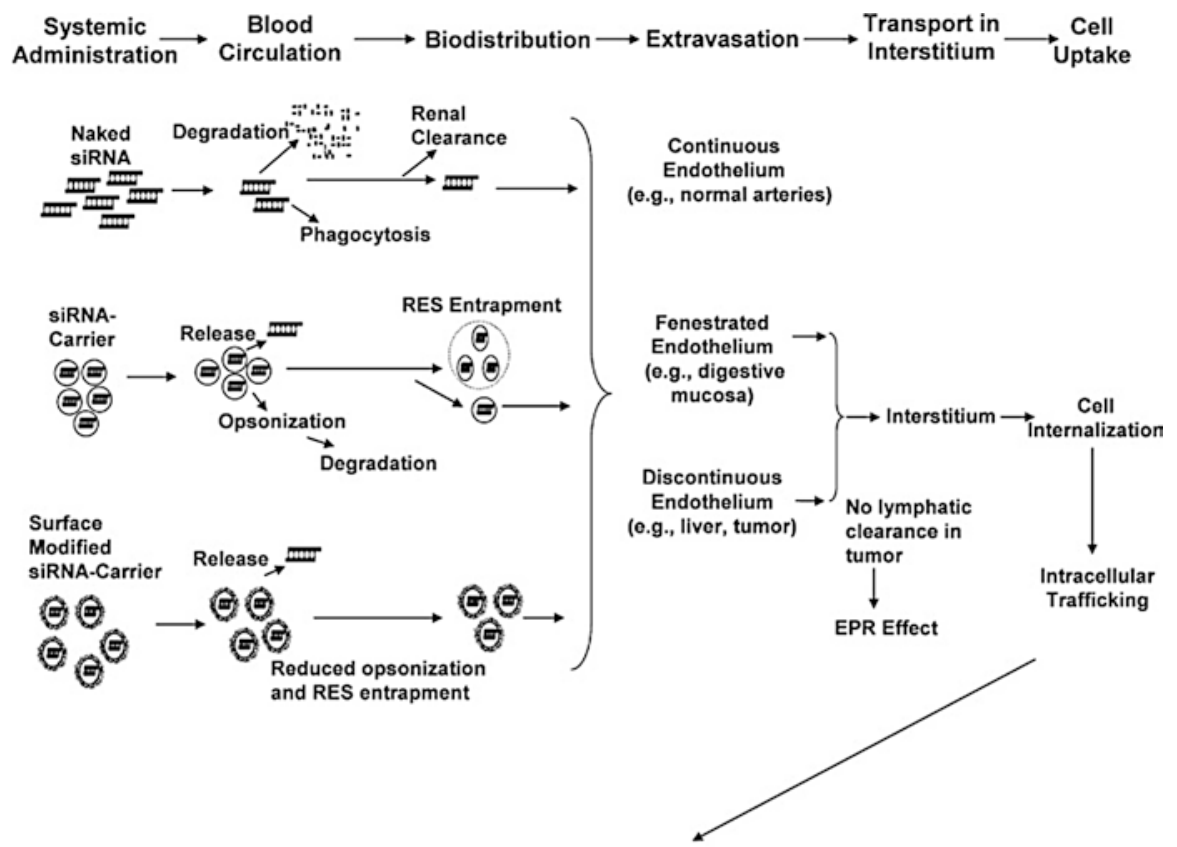

Intracellular Trafficking

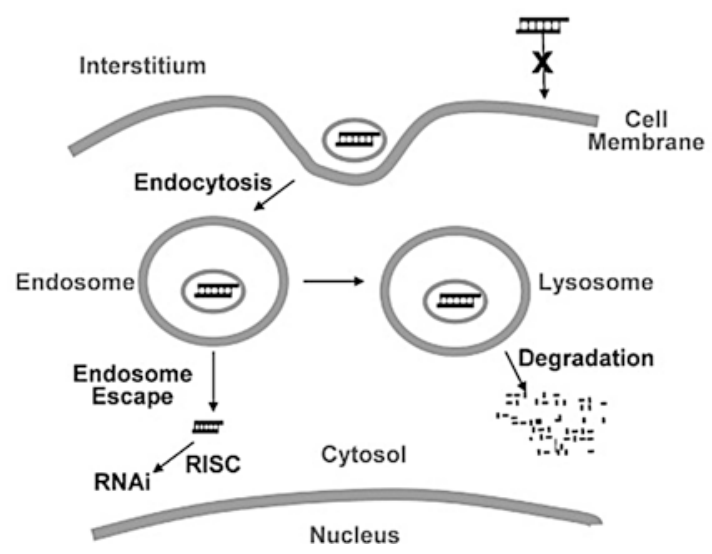

Fig. 3 Barriers to systemic siRNA delivery. (This figure is produced after reprint permission under license no. 4445840089493). (Wang et al. 2010)

RNA can perform their gene silencing function. The exposed siRNA molecule being negatively charged and large, constrain its own passage through the cell membrane and anticipates aggregation inside the cell.

The second barrier to overcome is endolysosomal compartments within the cytoplasmic space. These vesicles also contain various intracellular RNAase which may degrade the siRNA molecule once internalized i.e. endosomal engulfing. Strategies 
for designing and delivering siRNA are therefore essential for enabling endocytosis and subsequent endosomal escape of siRNA molecule. Additionally, siRNAs being susceptible to hydrolysis by endosomal nuclease enzymes can be incorporated into RISC with improved efficacy, and to compel the clinical utility (Gavrilov and Saltzman 2012).

\subsection{Off-Target Effect}

Recent studies conducted by Birmingham et al. (2006), Mouldy (2006), and Wilson and Doudna. (2013) have shown that the specificity of RNAi is sometimes compromised. The evidence from these studies shows that most siRNA molecules are probably not as specific as it was presumed and that siRNA treatment can bring about off-target gene silencing. The off-target effect refers to the suppression of genetic region other than the causative gene target. Such a phenomenon may produce undesirable genetic alteration and cellular dysfunction. The most common reason of "off-target" silencing is the seed region of the siRNA sequence. This region is characterized by partial homology of 6-7 nucleotide long sequence particularly inside the $3^{\prime}$ untranslated sequence ( $\left.3^{\prime} \mathrm{UTR}\right)$ which bound with a different mRNAs rather than the desired target mRNA (Birmingham et al. 2006). In the condition, a seed region siRNA acquiring complementarity with certain regulatory miRNAs may distort the gene expression (Mouldy 2006). Moreover, failure of discrimination between the guide strand and the passenger strand by RISC may prompt the chances of complementary interaction between siRNA and non-targeted mRNA. The offtarget effect can also arise as an after effect of the immune activation. RNA if perceived by immunoreceptors sometimes, such as Toll-like receptors (TLRs) and trigger signalling biomolecules such as cytokines which may alter gene expression (Wilson and Doudna 2013).

Off-target cleavage can't be overlooked as it prompts adverse mutations at the genetic level and results in unexpected consequences. Thus, the selection and sequencing of candidate siRNAs should be done specifically. Two basic processes can be followed for determining the potent RNAi sequence specific to the targeted gene. Predictive bioinformatic approaches implemented at the initial stage of siRNA to develop the most suitable sequences of the siRNA molecule. Later, exclusive trials on all potent therapeutic siRNA sequences should be performed to observe normal protein expression profiles. Any inadvertent off-target effect may result in an abnormal interpretation of data and potential toxicity. 


\subsection{Saturation of RNAi Machinery}

The bioactive material is usually analogues to the cellular biomolecules and works similarly to the in-vivo biomolecules. Externally delivered therapeutic siRNAs almost resembles intrinsic siRNAs and depend on the endogenous machinery for inducing RNA interference. Hence, there are significant risks of saturating such pathways and hence disturb the native biological systems that based on cellular processing to execute their role.

\subsection{Stimulation of Immune System by siRNAs}

While in general, the active immune mechanism serves for defence mechanism and is advantageous. However, sometimes, it evokes undesired immune sensitization, which may lead to adverse effects (Marques and Williams 2005). The siRNA might be discriminated as a foreign particle through various defence mechanisms and components of the immune system. For example, siRNA may be recognized by cytoplasmic and endosomal Toll-like receptors expressed on the surface of different immunocompetent cells. Consequently, the release of cytokines and type-I interferon generates inflammatory reactions. The immune stimulation property of siRNA is directly related to its size. siRNA oligonucleotide up to 30 base pairs usually doesn't confer cytotoxicity. A cell could safely be transfected with 30-nucleotide bp longer dsDNA without any cellular toxicity. On contrary, longer RNA strands elicit an immune response as it bounds to either TLRs or to certain enzymes such as $2^{\prime}, 5^{\prime}$-oligoadenylate synthetase-RNase L or protein kinase to trigger interferon. There are specific GU-rich motifs which are actually determined by TLR7, refer as "danger motif". For example, in the endosome vesicle of plasmacytoid dendritic cells 5'-GUCCUUCAA-3' sequence is recognized by the TLR7 and stimulates innate mechanisms to release inflammatory cytokines further. Another example is of TLR9 activity is countering CpG motifs in antisense oligonucleotides. Thus the immune actions can be controlled by mode of delivery and passage across various intracellular compartments of the siRNA. RNA oligonucleotides larger than 27-29 base pairs and highly potent Dicer substrates siRNAs need to be safely administered and monitored for immunogenic side-effects (Rose et al. 2005). 


\section{Overcoming the Hurdles to siRNA Delivery Using Nanocarriers}

To overcome the above-discussed therapeutics hurdles, and to achieve efficient and potent systemic delivery of unstable siRNA, several methods have been developed that can increase the stability in serum, improve in-vivo delivery and efficacy of gene silencing tendency. Some of them are-

(i) Designing artificially chemically modified siRNA structures which are prepared by substituting various molecules or atoms at different positions for examples-substituting phosphodiester $\left(\mathrm{PO}_{4}\right)$ linkages with phosphorothioate (PS) at the $3^{\prime}$-end, or introducing the new molecules that resist the enzymatic degradation like adding $\mathrm{O}$-methyl (2'-O-Me), fluoro ( $\left.2^{\prime}-\mathrm{F}\right)$ groups or methoxyethyl (2'-O-MOE) group significantly prolonged circulation half-life in systemic route and improved RNAi efficiency (Czauderna 2003; Chiu and Rana 2003).

(ii) By complexation with a molecule or carrier, for example, the cholesterolsiRNA complex. The cholesterol-siRNA complexes improve pharmacokinetics (increases half-life of siRNA) (Wolfrum et al. 2007).

(iii) By encapsulating siRNA in a carrier such as viral vectors. Various natural viral vectors have already in use for systemic transport of siRNA to treat various carcinomas. Jung et al. used an adenoviral vector encoding siRNA significantly suppressed the outgrowth of the pituitary tumor by transforming gene overexpressed in both in-vivo and in-vitro hepatocellular carcinoma cells (Jung et al. 2006). Sabbioni et al. (2006) showed type 1-based amplicon vectors that based on herpes simplex virus, inhibited in-vivo tumor formation of human polyomavirus BK-transformed cells (pRPc cells) (Sabbioni et al. 2006).

Even though the above-mentioned methods have several advantages and provide a good platform to improve delivery of naked siRNA. However, the frequent application of these methods is limited because they are also having numerous drawbacks such as safety issues (insertion mutagenesis and immunogenicity) low loading efficiency and only laboratory/small-scale production. Furthermore, siRNA is more liable to nuclease degradation than circular plasmids. Therefore, we need a stable delivery system to improve the systemic delivery of siRNA.

Now a day, an alternative approach, synthesis of nonviral vectors (synthetic carriers) is inflowing to resolve or reduce these drawbacks and to enhance in-vivo siRNA delivery. Nonviral vectors have various sizes, shapes, dimensions, and consist of different biocompatible and biodegradable materials. These carriers can load or entrap the high amount of siRNA and shielding from various hurdles or barriers such as rapid elimination, enzymatic degradation, reduced potency in-vitro and invivo both etc. Currently, different dimensions, shapes, nanosized (at least one dimension $<100 \mathrm{~nm}$ ) nonviral vectors are applied for the systemic delivery of genes, drugs, or co-delivery of both genes and drugs together. These nanosized, nonviral vectors enhance pharmacokinetics (increase half-life) with EPR (enhanced perme- 
ability and retention) effect, decrease rapid renal filtration/clearance, and protect from extracellular enzymatic degradation and support in escape from endosomes intracellular. These NCs have some beneficial properties like high biocompatibility, non-immunogenicity etc. Polymer, lipid-based nanomicelles liposomes, and dendrimers are mostly used nonviral NCs.

\section{The Aid of Nanocarriers for siRNA Delivery}

\subsection{Enhanced Blood Retention Time}

The size of naked siRNAs is about 7 in length (Hansen et al. 2001; Smith et al. 1996) and $2 \mathrm{~nm}$ in diameter (Sinden et al. 1998) and with a molecular weight of about $13 \mathrm{kDa}$ (Whitehead et al. 2009). The kidney is made up of numerous glomeruli, and the main function of glomeruli is the natural filtration barrier of undesired by-products in the blood. The size of pore the glomeruli membrane is approximately $8 \mathrm{~nm}$ (Wartiovaara et al. 2004). Any molecules that have a molecular weight and a size below $50 \mathrm{kDa}$ and $8 \mathrm{~nm}$, respectively are typically rapidly expelled from the kidney (Rappaport et al. 1995). After systemic delivery, siRNA is rapidly expelled out from the body system through either renal clearance and filtration through the renal system or the entrapment in the RES in the liver, spleen, lungs and bone marrow. For increasing the retention time of siRNA in the body, siRNA is entrapped indifferent NCs that have the size range between 20 and $100 \mathrm{~nm}$ in diameter. This optimal size range helps to avoid potency for avoiding both renal clearance and RES entrapment and allows NCs to remain in the circulation for a longer period and to increase the rate of intracellular penetration in tumor cells and tissues via passive targeting (Jang et al. 2003; Pecot et al. 2011).

\subsection{Enhanced Stability and Cell Penetration Property}

The specific physiochemical properties of naked siRNA are their anionic charge due to the presence of phosphate groups in the structure and hydrophilicity. As the plasma membrane is made up of lipid bilayers, it is lipophilic in nature and having anionic charge. Therefore, the intracellular penetration of siRNA across the plasma membrane is quite limited by passive transportation due to the electrostatic repulsion. When the siRNA is administrated in the bloodstream, it is immediately covered by a major component of blood plasma protein i.e. serum albumin and initiates our immune system to engulf albumin coated siRNA by phagocytes NCs entraps or covered the siRNA, avoid the engulfment by the phagocytes cells and increase the cell penetration of siRNA. Polycationic (positively charged) NCs are the best suited for high siRNA loading, shielding from degradation during bloodstream circulation, 
and systemic delivery of siRNA, especially to metastatic tumors. The most commonly used polymers are polyethyleneimine (PEI) (Illum and Davis 1984; Norman et al. 1993). According to Bonnet et al. (2008) linear PEI-siRNA (IPEI/siRNA) conjugate showed better cytocompatibility and non-immunogenic than branched PEIsiRNA (bPEI/siRNA) conjugate (Bonnet et al. 2008). However, the NCs that are prepared from a single PEI polymer is not stable during systemic delivery of siRNA and agglomerate in the bloodstream due to slat medium and serum protein. The prominent solution to overcome the problem is to use a copolymer/mixed polymer (di or triblock copolymers) which are more stable, for example, PEI can be mixed with poly (ethylene glycol) (PEG) to prepare a PEGylated PEI/siRNA. PEG polymer covers at the outside of the PEI/siRNA conjugate by forming a hydrophilic layer that sufficiently reduces the agglomeration tendency of NCs with increased EPR effect (Malek et al. 2008; Merkel et al. 2009). Kim et al. (2012) prepared mannosylated and pegylated polyethyleneimine/siRNA (PEI-PEG-MAN/siRNA) polyplex NCs with an approximate size of $357.33 \mathrm{~nm}$ for increased cellular uptake of siRNA. In this conjugate, PEI entrapped a high amount of negatively charged siRNA, PEG provided outer stable hydrophilic membrane, and mannose was used as a cell binding ligand for macrophages (Kim et al. 2012).

Some other polycationic peptides, like poly-histidine polymers, and lipids NCs have also used for the systemic and improved delivery of siRNA (Midoux et al. 2009).

\subsection{Site-Specific Delivery}

Although after entrapment of siRNA by different positively charged NCs enhance the therapeutic efficacy of siRNA, the delivery systems are not enough to deliver a high amount of siRNA to the specified tissues/cells by passive diffusion transport. Due to this, NCs are not capable to differentiate infected (cancer) cells from normal/ healthy cells. Also, positively charged NCs agglomerate in different cell media and biological fluids. To increase the concentration of siRNA on the targeted site, the nanocarriers-mediated targeted delivery system is a new and novel approach to increase the therapeutic efficacy of siRNA. Receptor-mediated endocytosis is an active transport system that improves the rate of cellular uptake of siRNA loaded NCs. The active, targeted transport system can be achieved by surface modification of NCs using different targeting ligands. These specific ligands bind to a site-specific receptor which is overexpressed on targeted cells and tissues ( $\mathrm{Li}$ et al. 2016; Akinc et al. 2010). Examples of some commonly used ligands are (poly)saccharides, (Zhang et al. 2016) vitamins, (Duan et al. 2016) antibodies, (Gu et al. 2017) peptides, aptamers, transferrin (Tf) etc. 


\section{4 pH-Sensitive Trigger Release}

The development of pH-sensitive NCs is the most commonly used approach to improve delivery of siRNA after systemic administration, especially in cancer. The $\mathrm{pH}$ of blood, extracellular matrix and fluid are mildly basic $(\mathrm{pH} \sim 7.4)$, whereas the $\mathrm{pH}$ of the intracellular compartment is 6.8. However, cancer cells have even more acidic ( $\mathrm{pH} 4-6)$ for both extracellularly and intracellularly, caused by the higher rate of glycolysis and tendency to produce higher $\mathrm{CO}_{2}$ and lactic acid. High lactate levels indicate metastases, tumor recurrence, and prognosis in some cancer patients. Basically, there are three different materials are used to develop pH-sensitive NCs: protonizable, acid-labile, and destabilizing compounds (Torchilin 2014; Sawant and Torchilin 2012).

Poly(2-(diisopropylamino)ethyl methacrylate) (PDPA) is a smart polymer, that is used to form NCs. These NCs bypass the endosomal compartment and improve the efficacy of siRNA delivery (Qian and Berkland 2015). It is a pH-sensitive polymer that is hydrophobic in nature at neutral and physiological $\mathrm{pH}$ medium. However, in mild acidic medium $(\mathrm{pH}<6.2)$ it changes from hydrophobic to hydrophilic in nature (Licciardi et al. 2005). This polymer can easily encapsulate the desired amount of siRNA and shield the siRNA from enzymatic degradation at physiological $\mathrm{pH}$. The encapsulated siRNA is released in the cytoplasm after dissociation of NCs in early endosomes or lysosomes $(\mathrm{pH}<6)$ via protonation of the PDPA and display an outstanding 'proton sponge' effect (PSE) due to tertiary amines at the terminal surface (Yu et al. 2011).

Numerous NCs have been developed that are stable at blood $\mathrm{pH}$ and give the protection of siRNA during circulation in the bloodstream but they degrade at low $\mathrm{pH}$ in tumor tissue environment and release the siRNA (Kim et al. 2012).

PSE is the effect that is used for protecting the degradation of therapeutics agents loaded NCs from different cell organelles such as early endosome and lysosome using various aminated polymers like PDPA, PEI, (Benjaminsen et al. 2013) polyL-Lysine (PLL) etc. These polymers are destroyed to cell organelles upon acidification in low or acidic $\mathrm{pH}$ and safely deliver the genetic material in the nucleus. The PSE produces from numerous slightly basic cojugates (between $\mathrm{pH} 5$ and 6), promoting proton $\left(\mathrm{H}^{+}\right)$absorption in cellular organelles with lower $\mathrm{pH}$ and an osmotic pressure generated around the organelle membrane. Due to the generation of osmotic pressure, acidic endosome burst and release the materials into the cytoplasm. The advantage of the PSE is that we could easily control this effect by partially altering the groups from carboxylic acid $(-\mathrm{COOH})$ to tertiary $\left(3^{\circ}\right)$ amines. Whenever both the functional groups are attached on the surface of NCs, form electrostatic and steric interactions that are extremely reactive to the acidic organelles and siRNA is appropriately attached and cellular entry (Yezhelyev et al. 2008). 


\subsection{Avoid Intracellular Endosomal Engulfing}

As discussed earlier, naked siRNA cannot efficiently across the plasma membrane due to unfavorable physicochemical properties of siRNAs. A very less amount of siRNA is passed through from the semi-permeable plasma membrane. After the intracellular delivery, siRNA is less stable in the bloodstream and has a very less half-life $(<5 \mathrm{~min})$ due to rapid enzymatic degradation by endogenous serum nucleases (Tseng et al. 2009). The intracellular trafficking of siRNAs starts in early endosomal vesicles where siRNA fuse with sorting endosomes and finally transfer their contents to the late endosomes. A high amount of siRNA is entrapped by the late endosomes and lysosomes and very less amount of siRNA is reached into the cytoplasm to come into play regardless of the release mechanism. To avoid the rapid degradation and shielding from engulfing by the late endosomes and lysosomes, various NCs have been developed to shield the naked siRNA such as cationic lipid, (Lu et al. 2009) polymers and di or triblock copolymers NCs, (Qian and Berkland 2015) nanomicelles (Zhou et al. 2016) etc.

\section{Classification of Nanocarriers Used for Systemic Delivery of siRNA}

The NC that is applied to shield and to improve in-vitro and in-vivo delivery of siRNA must be safe and non-toxic for human health because they come in direct contact with humans. Naturally obtained molecules are safer as compared to chemically synthesized molecules. Because of the synthesis of NCs several hard acids and bases, and others hazardous chemical are used. These acids and hazardous chemicals give several toxic effects on human health and the environment also. Mostly NCs have the diameter $>100 \mathrm{~nm}$. However, the long chain polymeric NCs can have the diameter $>500 \mathrm{~nm}$. NCs developed for the systemic delivery of siRNA can be broadly classified into three different types:

\subsection{Organic Nanocarriers}

Organic nanocarriers (ONCs) are prepared from natural or synthetic organic molecules and the mixture of both. ONCs are typically soft, biocompatible, biodegradable, less toxic, non-immunogenic, highly stable, and can shield the siRNA. From the last few decades, several scientists and researchers have already used numerous organic NCs for protecting the drugs and nucleic acids from the harsh environment, enzymatic degradation and blood plasma proteins during systemic circulation inside 
the body. The most commonly used ONCs are nanomicelles, liposomes, polymeric nanoparticles, dendrimers, and carbon nanomaterials (graphene oxide sheets, nanotubes, spherical shape fullerene). These NCs have some additional benefits. The synthesis procedures for ONCs are quite easy and they can encapsulate more drugs or nucleic acids molecules with a controlled delivery. The surfaces of ONCs can be simply decorated with targeting biomolecules to develop targeted carrier systems. These advantages make ONCs the most appealing systems for drugs and genes delivery and other biomedical applications.

\subsubsection{Polymeric Nanocarriers/Polyplexes}

Currently, several natural and synthetic polymeric nanocarriers (PNCs) (Fig. 4c) offers a great platform to deliver drugs and gene with improved efficacy. These polymers are highly biocompatible, biodegradable, non-toxic in nature and have outstanding controlled release character. Generally, PNCs are cationic, anionic and non-ionic in nature. The polycationic polymer has emerged as one of the most promising candidates for developing efficient gene delivery vector.

Polymers can be broadly classified into two-types based on their sources: natural and synthetic. The most commonly used natural polymer is chitosan (CS), poly(lactic acid-co-glycolic acid) (PLGA), (Zhou et al. 2012) atelocollagen, (Minakuchi et al. 2004) inulin (Cavallaro et al. 2017). CS is a linear, natural, cationic, FDA (Food and Drug Administration) approved polysaccharide (a long chain of monosaccharide carbohydrate) co-block polymer of glucosamine and $\mathrm{N}$-acetylated glucosamine. Polymer and has been used for both in-vitro and in-vivo systemic delivery of siRNA because it has nontoxicity, biocompatibility, and biodegradability properties (Malhotra et al. 2013; Vauthier et al. 2013). Currently, CS is the highly used polysaccharide polymer for delivering of therapeutic agents (drugs and genetic agents) due to its high permeability capability across the semipermeable cell membrane (des Rieux et al. 2006). In one of the study, prepared an novel amino acid-functionalized Arg-Gly-Asp (RGD)-chitosan nanoparticle (RGD-CHNP) that significantly increased selective intratumoral delivery of siRNA for regulation of many growthpromoting genes (PLXDC1, FAK, and POSTN) accompanied by therapeutic efficacy in the A2780, HeyA8, and SKOV3ip1 orthotopic animal studies of ovarian cancer models (Han et al. 2010). Besides natural polymers, several biocompatible, biodegradable, non-toxic synthetic PNCs have also been used for systemic delivery of siRNA such as PLL (Cun et al. 2011), PEI (Nimesh and Chandra 2009), PEG, dimethylamino ethyl methacrylate, polyfluorene, and cyclodextrin-based polycations. These polymers are linear and branched and similarly the efficient way to shield and efficient delivery of siRNA. Both types of PNCs can encapsulate a high amount of siRNA and deliver it via the passive route. For increasing the delivery concentration of siRNA on specific infected cells/tissues by escaping the normal cells and tissues, different actively targeted molecules are attached to the surfaces of PNCs (Table 1). 


\section{Organic Nanocarriers}
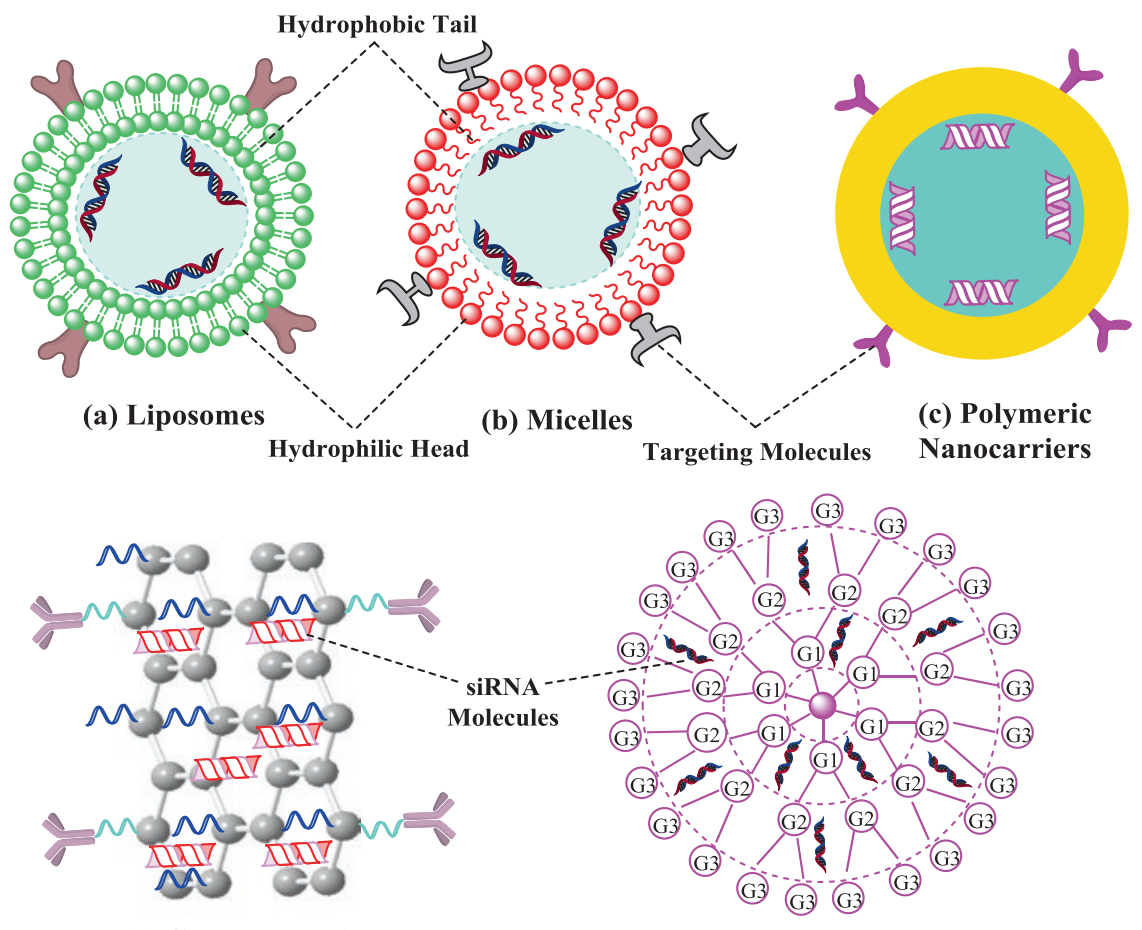

(d) Graphene Oxide

(e) G3 Dendrimer

Inorganic Nanocarriers

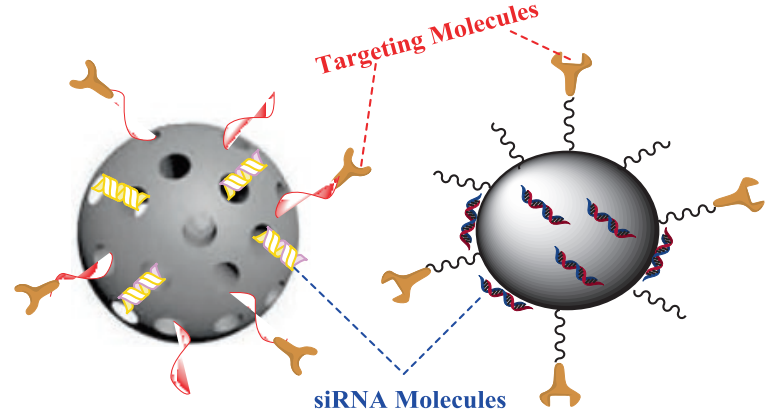

(f) Mesoporous

Silica Nanoparticles (g) Iron Oxide Nanoparticles

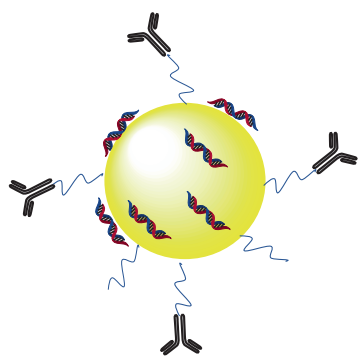

(h) Gold Nanoparticles

Fig. 4 Schematic illustration of various nanocarriers 


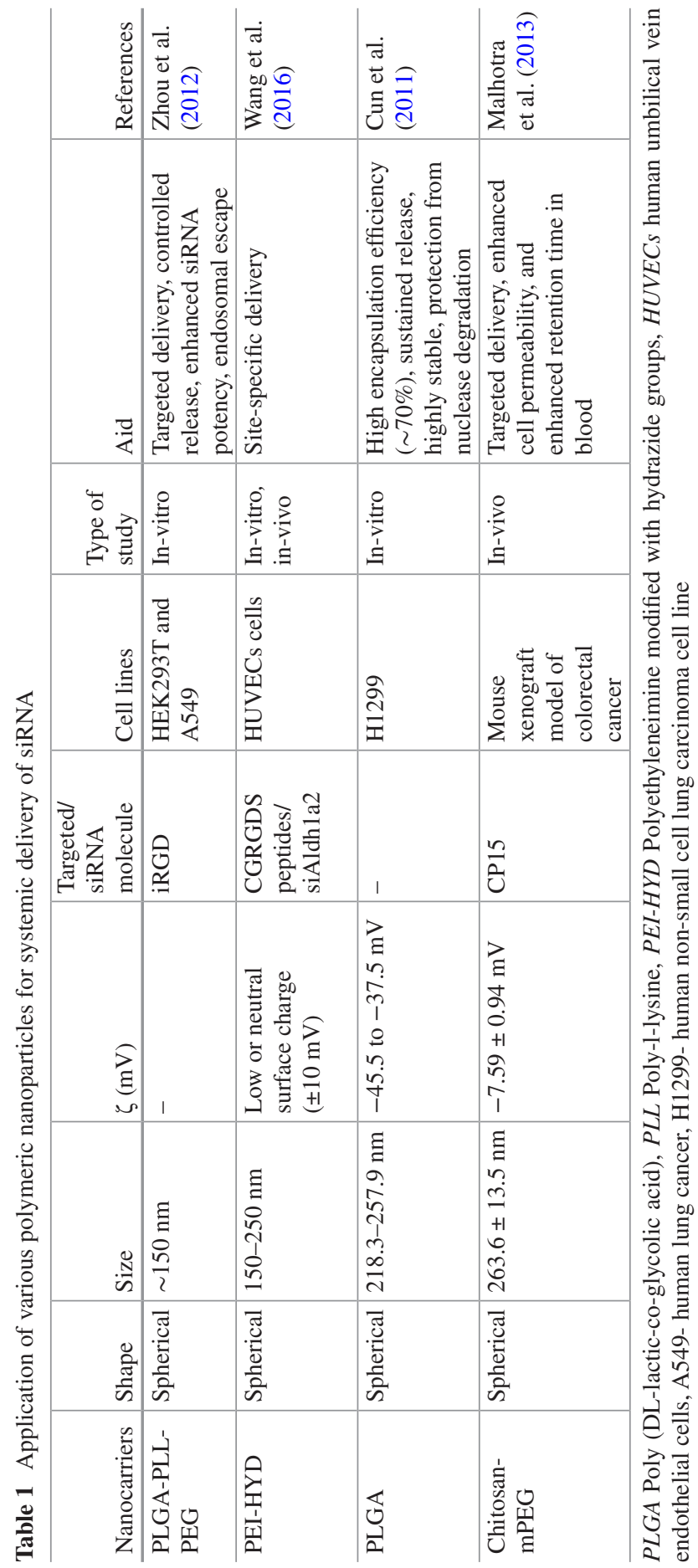




\subsubsection{Nanomicelles-Based Delivery}

The term "micelles" was given by McBain in 1913. Micelles are formed by supramolecular self-assembly of surfactants, lipids, aqueous insoluble polymers (Euliss et al. 2006). Amphiphilic molecules are the molecules that have hydrophobic nonpolar "tail" at one end and hydrophilic polar "head" to the other end (Fig. 4b). Micelles are the colloidal suspension of the amphiphilic molecules. When amphiphilic molecules are mixed in the aqueous medium, the nonpolar hydrophobic tail is arranged inside the molecules and letting the polar side i.e. "head" to remain an outer side in direct contact with the aqueous medium and a hollow spherical or cylindrical structure is formed by the process of self -assembly. For example, Cao et al. (2011) mixed cationic PCL and PEI which self-assembled, forming biodegradable micelles. These micelles were utilized to carry anti-apoptotic Bcl-2 specific siRNA and along with an anti-cancer drug, docetaxel (DOX). Further, folic acid was surface-conjugated as a targeting ligand for hepatic cancer cells. This nano-assembly simultaneous delivered drug and siRNA to yield a combined RNAichemotherapeutic benefit against hepatic cancer (Cao et al. 2011). Similarly, Zhu et al. (2014) synthesized PEG-pp-PEI-PE nanomicelles for the delivery of siRNA against survivin or GFP along with paclitaxel (PTX). Efficient down-regulation of GFP gene and survivin were observed in PTX-resistant non-small cell lung cancer cells after the micelles-mediated siRNA delivery compared to free drug (Zhu et al. 2014). In another study, prepared a PEGylated PEI-siRNA micellar nanoparticles that shown high entrapment efficiency and long-term blood circulation of siRNA loaded nanoparticles with reduced aggregation, opsonization, and inflammation response. In this study, polycationic PEI was used for high loading capacity of negatively charged siRNA and PEG was used for others advantages (Wu et al. 2017) (Table 2).

\subsubsection{Carbon-Based Nanomaterials}

Carbon nanomaterials (CNMs) are synthesized from allotropes of carbon such as graphene, graphene oxide (GO), nanotubes, fullerene. For example, a study conducted by Varkouhi et al. (2011), carbon nanotubes functionalized with PEI and pyridinium were explored for siRNA delivery. Both functionalized cationic CNTs complexed anionic siRNA and provide the silencing efficiency of 10-30\% and 10-60\% of cytotoxicity (Varkouhi et al. 2011). Another study by Ren et al. (2017) was done to prepare and characterize a GO-based carrier, surface-functionalized with PLL and Arg-Gly-Asp-Ser oligonucleotides to actively target tumors, These nanocarriers were then used for loading VEGF-siRNA and observed to perform a slow and sustained release of siRNA along with low toxicity as compared to bare GO (Ren et al. 2017).

Figure 4d showed the two-dimensional, sheet-like GO structure that has numerous oxygen groups like carboxylic acids, hydroxyl, epoxy, and alkoxy on its edges and both side of surfaces. The average thickness of GO is about 1-2 nm and length/ 


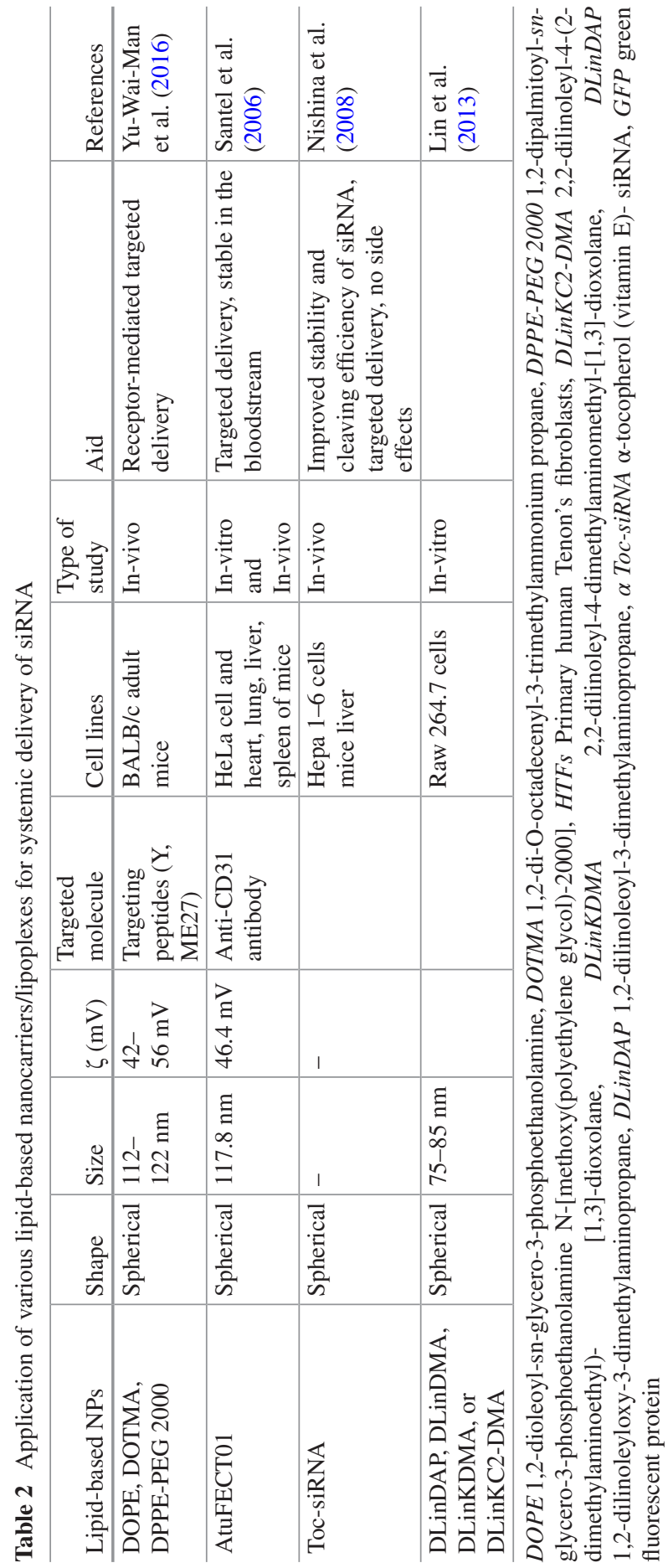


width is between 50 and $200 \mathrm{~nm}$ depending on the biomedical application. GO has a high surface area and capability of holding molecules on both sides of sheets due to this GO has high loading capacity.

Carbon nanotubes (CNTs) are hollow one-dimension nanostructures. The CNTs may be cylindrical, tubular or needle in shape. Depending on the number of layers/ walls, CNTs are of three-types: single-walled carbon nanotubes (SWCNTs), double-walled carbon nanotubes (DWCNTs) and multi-walled carbon nanotubes (MWCNTs). The typical thickness of SWCNTs is about $0.4-1 \mathrm{~nm}$ and that of DWCNTs is 1.4-20 nm (Schedin et al. 2007; Bae et al. 2010). MWCNTs are 20-100 nm thick (Bartelmess et al. 2015) and 50-500 nm in length.

Fullerene has zero-dimensional foot-ball like hollow structure with a diameter below $100 \mathrm{~nm}$. Fullerene has a different number of carbon atoms on its structure like $\mathrm{C}_{60}, \mathrm{C}_{70}$ etc. $\mathrm{C}_{60}$ is the most commonly used fullerene, having 60 carbons in its structure and is composed of 20 hexagonal rings and 12 pentagonal rings and is called as Buckyballs.

Pristine CNMs are less biocompatible and have less siRNA loading capacity due to electrostatic repulsion between them. CNMs are modified or coated with several cationic polymers like PEG, PEI, chitosan, PAH (Poly (allylamine hydrochloride)), PLGA (Poly (lactic acid-co-glycolic acid)) are the frequently used polymers. After modification or coating with the polymer, CNMs can hold a high concentration of siRNA due to the simple electrostatic attraction between of them. For improved delivery of siRNA, polymer coated CNMs are further modified with suitable ligands (Table 3)

\subsubsection{Liposomes/Lipoplexes}

Liposomes are well-ordered, self-assembled vesicles of amphiphilic lipids molecules that form a minute spherical shape nanoparticles having single-lamellar or multi-lamellar lipid bilayer structure of phospholipid molecules that have aqueous medium inside used to encapsulation and deliver drugs or genetic materials into a cell (Fig. 4a) (Akbarzadeh et al. 2013). Liposomes are highly biocompatible and biodegradable, small size, vesicles of amphiphilic lipid with the high surface areato-volume ratio. They have some superior physiochemical properties like increased rate of biological membrane permeability, the flexibility of functionalization, tuning of surface charge. These properties make liposomes a better nanoformulation to deliver drugs and genes than others NCs and also have been successfully applied in the clinic (Hatakeyama et al. 2011). Polycationic liposomes as building blocks to entrap of negatively charged siRNA through electrostatic interactions and form lipid complexes systems called lipoplexes (Wolff and Rozema 2008). So far, many liposomal formulations had got the FDA approval and many more are currently in development or in clinical trials enabling features such as stable circulation and tumor-specific targeting versions of siRNA-liposome carriers (Noble et al. 2009; Drummond et al. 2010; Patil and Jadhav 2014). Liposomal NCs can be divided into 


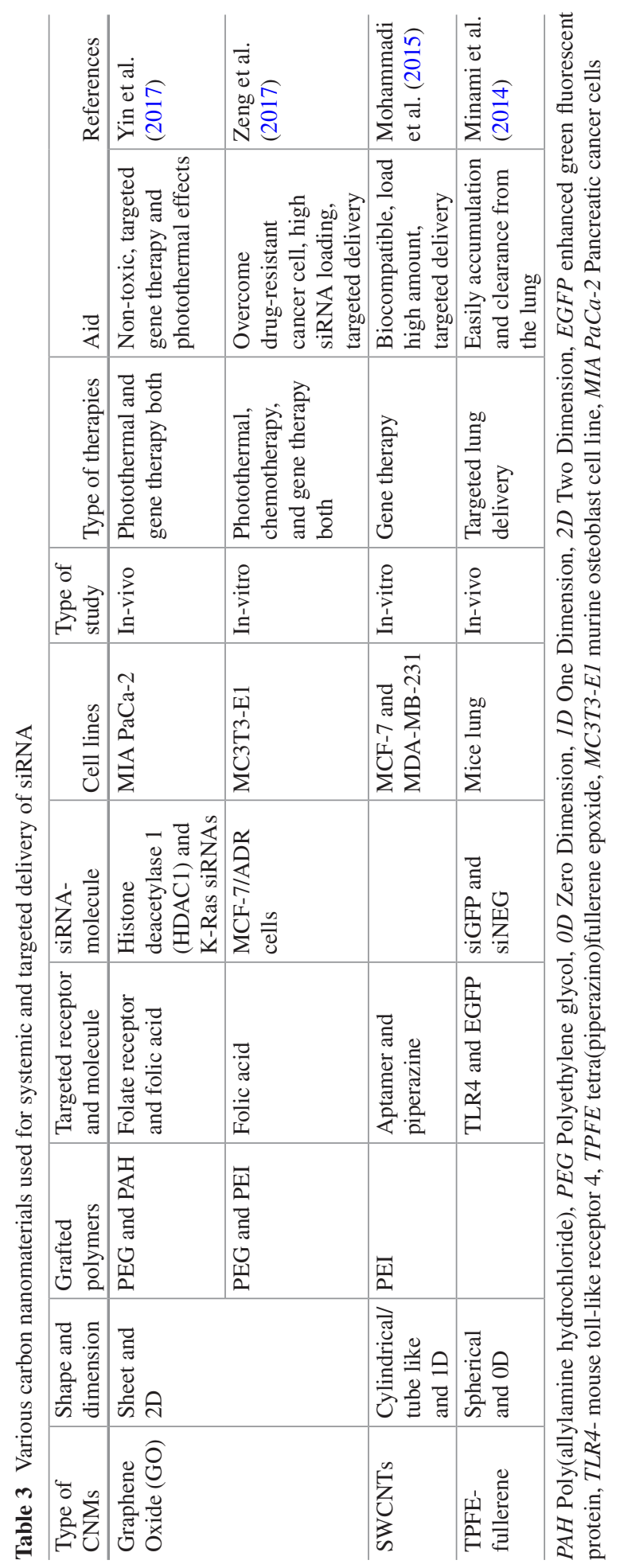


four main types based on their physical structures- nucleic-acid-lipid particles, lipoplexes, lipopolyplexes, and membrane/core nanoparticles.

Lipoplexes are composed of several bilayer of lipids in spherical form and siRNAs are entrapped between the consecutive lipid bilayers. Stable nucleic-acid-lipid particles have the outer surface is almost neutral but inner surface are highly positively charged due to this inner positive charge siRNAs are entrapped in the interior of liposomes. Only pH-sensitive lipid can be synthesized by these types of liposomes. These liposomes showed cationic behaviour at acidic $\mathrm{pH}(\mathrm{pH}-4.0)$ and noionic behaviour at neutral $\mathrm{pH}$ (pH-7.4). Lipopolyplexes are synthesized by coating the liposomes from oppositely charged polymers. If lipid NCs has cationic charged then they are coated with anionic charged polymers and vice versa. A membrane/ core NCs has some different structure than other liposomes NCs. This type of NCs is similar core-shell-like entities in which single or multiple inorganic NPs are embedded in the core and surrounded by a lipid bilayer form as the outer shell (Xia et al. 2016).

Landen et al. (2005) siRNA was encapsulated into 1,2-dioleoylsn-glycero3-phosphatidylcholine (DOPC, a neutral lipid) lipid NCs and this NCs were decorated with EphA2 (oncoprotein, a targeting ligand) for improved systemic siRNA delivery. These liposomal NCs was tested on two different orthotopic mouse model ovarian malignant cell types HeyA8 and SKOV3ip1. EphA2-siRNA-DOPC NCs have significantly reduced the tumor growth compared to non-silencing siRNA. Whenever paclitaxel was also loaded on this NCs conjugate the cancer cell growth was drastically decreased on comparison to individual chemotherapy with paclitaxel and RNAi therapy with a non-silencing siRNA (Landen et al. 2005).

\subsubsection{Dendrimers}

Dendrimers are the nanosized, spherical, monodispersed, highly branched, threedimensional, synthetic macromolecules. A typical dendrimer molecule is composed of three structural regions: the innermost core, comprising of atomic or molecular entity with at least two identical functional groups; branching generations with inner cavities, and specific functional groups present on the surface (Fig. 4e) (Verma et al. 2012; Pooja et al. 2014). Numerous functional groups (-COOH or $-\mathrm{OH})$ are suitable for the attachment of various ligands that makes it more suitable for targeted delivery of therapeutic agents. Dendrimers are made up of repeated branching unit and form a shell of either same groups and different groups, this shell is known as generation "G" (Kulhari et al. 2015). When are mixed with dendrimers known as dendriplexes. Presently, dendrimers are used for the delivery of several hydrophobic and hydrophilic drugs, genes, bioimaging agents (Table 4). Depending on the type of central atom, different classes of dendrimers are available such as PAMAM (Polyamidoamine), PPI (Poly (propylene imine), PLL, and poly(2,2bis(hydroxymethyl)propionic acid, etc. (Svenson and Tomalia 2012). Due to its branch structure, several voids/spaces are formed where drugs or nucleic acids are 


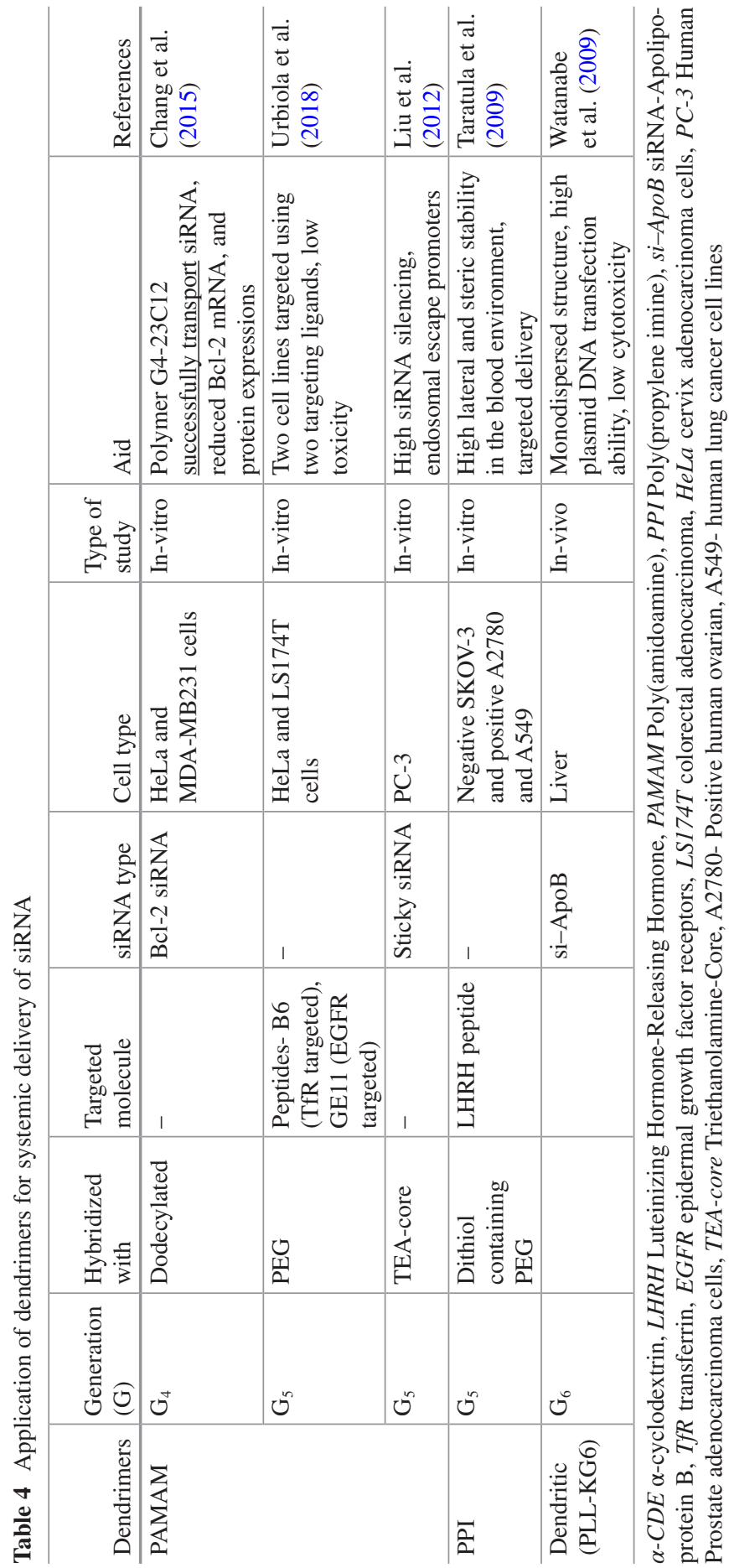


entrapped in these voids. Amine-terminated cationic dendrimers are good for high loading efficiency and controlled release of negatively charged siRNA.

Recently, for treating cancer researchers developed a tumor microenvironmentsensitive polypeptides-amphiphilic dendrimer as a carrier of siRNA/Paclitaxel (TMSP-ADENS/siRNA/PTX) complex for robust co-delivery of therapeutic agents. In this complex, siRNA and paclitaxel were encapsulated within the hydrophilic centre and internal hydrophobic layer, respectively. The outer PEG layer increased the half-life of dendrimers. This complex illustrated the highest rate while regulation of VEGF mRNA in A375 xenograft mice. Furthermore, the tumour-inhibiting and angiogeny-inhibiting activity were also observed in another study on HT-1080 xenograft (Li et al. 2018).

\subsection{Inorganic Nanocarriers}

Inorganic NCs are prepared synthetically from inorganic materials which are hard, water-insoluble, less biodegradable, toxic (Tatiparti et al. 2017). Due to these limitations, inorganic NCs are less used than ONCs to deliver the siRNA molecules. Frequently used inorganic NCs are mesoporous silica nanoparticles; different metal NPs such as gold NPs (AuNPs), selenium NPs (SeNPs), silver NPs (AgNPs); metal oxide NPs like super-paramagnetic iron oxide nanoparticles (SPIONs). Bare inorganic NCs are less biocompatible and have low siRNA loading capacity than ONCs. The reason is that these NCs are mainly solid and therefore they are unable to encapsulate any molecule. The molecules can only be adsorbed of any molecules on its outer most surface. For increasing their biocompatibility and loading capacity outer surfaces of these NCs are coated with several biocompatible, biodegradable, natural or synthetic polymers and lipids (Table 5).

\subsubsection{Mesoporous Silica and Silicon-Based Nanoparticles}

The use of mesoporous silica nanoparticles (MSNs) is increasing in biomedical and pharmacy field. MSNPs are not only having a spherical shape, high surface area, tunable small size, biocompatibility, and biodegradability, easily surface functionality but also have several ordered porous structural surfaces. Therapeutic hydrophobic drugs, bioimaging agents, genes, chemotherapeutic agents are trapped or stored in these pores (Fig. 4f). Due to this specific character, MSNs show some advantages of high loading efficiency and controlled release of encapsulated agents (Mekaru et al. 2015). Further, MSNs are small in size with a diameter ranging between 50 and $200 \mathrm{~nm}$, therefore they are passively transported and across membrane barrier and give EPR effect through and leaky vasculature and accumulate in the tumor cells (Maeda 2001). Homogenous, monodispersed MSNs are mostly synthesized by the sol-gel method. For high loading and specific site of delivery of anionic siRNA, 


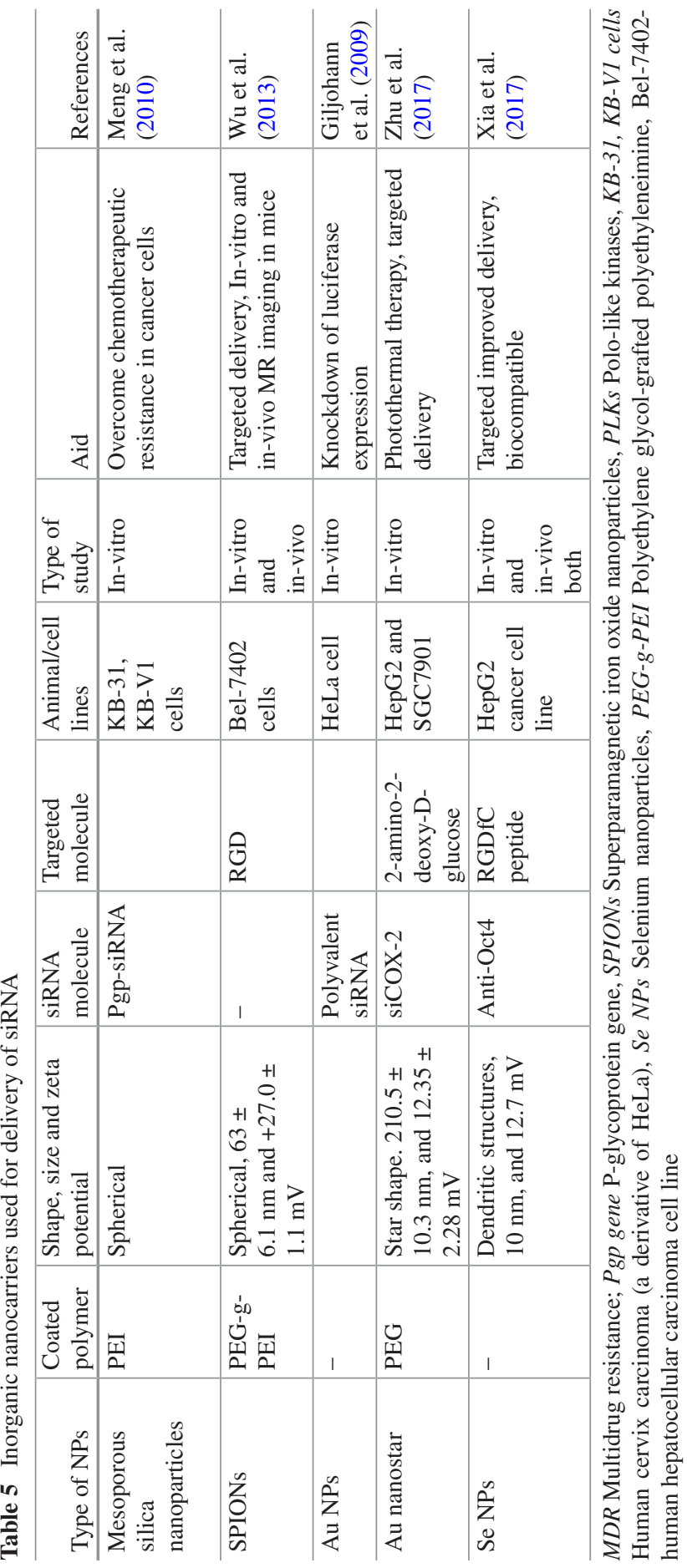


MSNs are coated with cationic polymers, PEG, PEI, and amine-terminated PAMAM dendrimers (Meng et al. 2010; Ngamcherdtrakul et al. 2015).

\subsubsection{Calcium Phosphate Core-Shell Nanocarriers}

Calcium phosphate $(\mathrm{CaP})$ particles are most commonly used biocompatible, biodegradable inorganic carrier for high loading of siRNA. Cap is a component that is also present in human bones. CaP particles have several demerits also they are obtained in micro-sized with polydispersed. Nano-sized monodispersed CaP NPs was obtained, when they were precipitated with PEG. CaP NPs are not shown any toxicity at a higher concentration than RNAi-induced concentrations and also shown better control over endogenous VEGF mRNA expression in cultured pancreatic cancer lines.

Researchers synthesized lipid/calcium/phosphate core-shell nanoparticles (LCP NPs) by microemulsion technology for improving systemic delivery of anti-luciferase siRNA. In this type of NPs, calcium phosphate core is surrounded by cationic lipid shell and decorated by an anisamide ligand complementary for sigma receptor on B16F10 melanoma cell surface. After IV injection of siRNA, the luciferase luminescence signal in metastatic B16F10 tumor cells was significantly diminished in C57BL/6 mice (Yang et al. 2012). Several others studies have also been done for systemic delivery of siRNA using CaP NPs inorganic nanoparticles (Qiu et al. 2016b; Pittella et al. 2011).

\subsubsection{Metal and Metal Oxide Nanoparticles}

From the last few decades, scientist and researchers have developed, tested and applied several types of metals (gold, silver, selenium, cobalt, nickel etc.) and metal oxides (iron oxide, silver oxide, manganese oxide etc.) NPs for the biomedical applications.

Initially, Au NPs were used only for drug delivery, biomolecular sensing, and hyperthermal therapy. However, currently, AuNPs are also being used in intracellular gene delivery and therapy due to their unique and controllable optical properties, and easy alteration of surfaces with thiolate molecules (Fig. 4h). Giljohann et al. (2009) prepared the polyvalent siRNA-gold nanoparticle conjugate (siRNAAuNPs) which was more stable in $10 \%$ serum, exhibited a prolonged half-life and more knockdown of luciferase gene expression than to free RNA duplexes (Giljohann et al. 2009). There are several ways to load a sufficient amount of siRNA on Au NPs:

(i) Thiol bond- siRNA is directly attached on AuNPs surface via a gold-thiol (R-S-H) bond.

(ii) Ionic bond- Anionic siRNA can be attached on the surfaces of the cationic AuNPs due to the electrostatic interaction. 
(iii) Polymer coated surface- siRNA adhered to the AuNPs surface coated with biocompatible and biodegradable with polymers (Lee et al. 2008).

Iron oxide NPs are other frequently used metal nanoparticles and have numerous biomedical applications. It is frequently used to deliver various therapeutic agents. Iron oxide NPs are also known as magnetic nanoparticles (MNPs) because they have high magnetic behaviour and this behaviour is used for remotely-controlled site-specific delivery of several therapeutic molecules (drugs and genes) by applying an external magnetic field (Fig. 4g). MNPs have a diameter between 10 and $100 \mathrm{~nm}$ range and are obtained in two different oxidation states- $\mathrm{Fe}_{2} \mathrm{O}_{3}$ (ferric oxide/ hematite) and $\mathrm{Fe}_{3} \mathrm{O}_{4}$ (magnetite/lodestone) NPs. When the diameter of NPs are $<30 \mathrm{~nm}$, they show the property of paramagnetism in the hysteresis of the magnetization loop of the particles because of the negligible coercivity arising from the infinitely small energy barrier (Lian et al. 2003).

\subsection{Hybrid Nanocarriers}

Organic and inorganic NCs are having their individual benefits and have been applied to remove various hurdles in delivering genetic materials. However, both classes of NPs has some limitations. Inorganic NPs such as MSNs may have more stability, mechanical, chemical and imaging properties but accumulates as nondegradable substances and confers toxicity. On the other side, organic NCs such as polymers, liposomes, and micelles are biocompatible as well as biodegradable with high encapsulation efficiency both on the surface and within the core, but less stable in-vivo. For removing the drawbacks of both type of NPs, the hybrid NCs are the best solution. These hybrid NCs are prepared with the fusion of both organic and inorganic NCs. Numerous biocompatible and biodegradable polymers are coated or grafted on the surfaces of both NCs. These polymers increase biocompatibility, loading tendency of genes with reduced toxicity (Tatiparti et al. 2017). Meanwhile the inorganic constituents provide diagnostic or therapeutic properties. Therefore, the hybrid NCs have benefit to offers the theranostics application with high delivery efficiency (Sailor and Ji-Ho 2012; Young et al. 2016) (Table 6).

\section{Conclusion}

Conventional therapy is already being applied to treat several deadly diseases such as cancer, tuberculosis, AIDS etc. However, their use is quite limited due to several weaknesses like non-targeted delivery, high dose and dose frequency that gives numerous adverse effects and multidrug resistance. Currently, gene therapy acclaimed as a revolutionary option of new therapy. siRNA delivery is an alternative approach, that has been pursued actively because of their excellent safety, high 


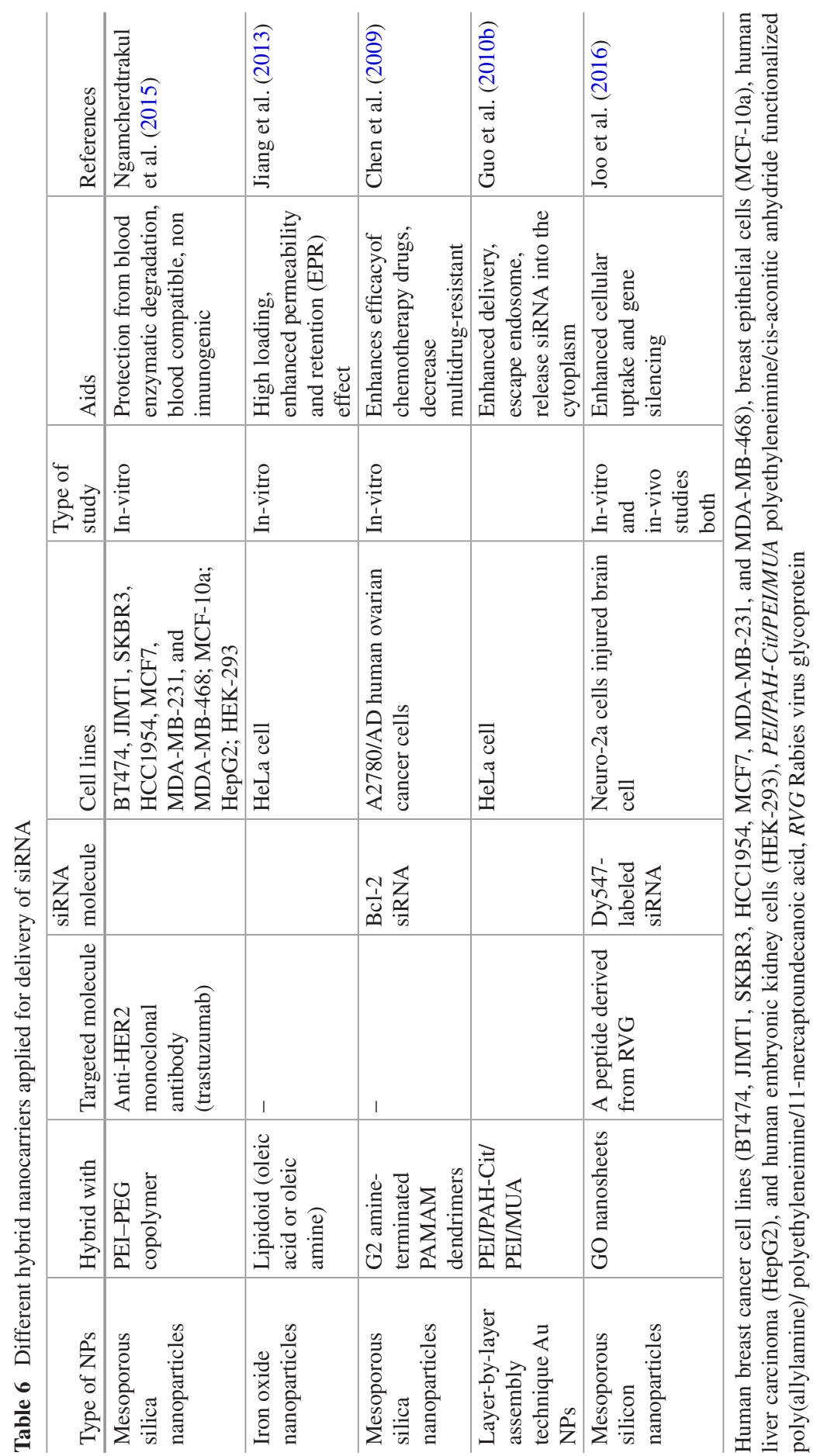


efficacy and specificity, easy modification, and unlimited therapeutic targets. But the therapeutic efficacy of naked siRNA is reduced due to systemic instability, before reaching to the target site. To get rid of these hurdles, the most vital solution is to develop biocompatible, biodegradable, and site-specific nano-sized carriers. Every NC has its own benefits and limitations depending on its physicochemical composition, offering a flexible and wide variety of effective agents based on siRNA. Organic NCs such as polymeric or lipid-based nanoparticles and micelles have excellent tendency to encapsulate the therapeutic molecules nevertheless they are unstable and easily degrade within the systemic circulation causing the premature release of therapeutic molecules before reaching the specific site. Mixed polymer micelles are quite stable compared to single polymer micelles. Inorganic naked metal and metal oxide NCs like Au NPs and MNPs are stable and can be easily functionalized. In spite of these advantages, several studies have proved that larger size and a higher concentration of these NCs are toxic. Moreover, naked NCs are also unstable in systemic delivery and rapidly agglomerated by blood plasma protein. Hence, these NCs require stabilizing materials. At last, the nanocarrier mediated delivery systems better solution to resolve issues related to the potent systemic delivery of siRNA. Some of the delivery systems also have proven their values and passed the initial clinical phases.

\section{References}

Aagaard, L., \& Rossi, J. J. (2007). RNAi therapeutics: Principles, prospects and challenges. Advanced Drug Delivery Reviews, 59(2-3), 75-86.

Ahmed, Z., Kalinski, H., Berry, M., Almasieh, M., Ashush, H., Slager, N., Brafman, A., Spivak, I., Prasad, N., Mett, I., et al. (2011). Ocular neuroprotection by SiRNA targeting caspase-2. Cell Death \& Disease, 2, e173.

Akbarzadeh, A., Rezaei-Sadabady, R., Davaran, S., Joo, S. W., Zarghami, N., Hanifehpour, Y., Samiei, M., Kouhi, M., \& Nejati-Koshki, K. (2013). Liposome: Classification, preparation, and applications. Nanoscale Research Letters, 8(1), 1-8.

Akhtar, S. (2009). Oral delivery of SiRNA and antisense oligonucleotides. Journal of Drug Targeting, 17(7), 491-495.

Akinc, A., Querbes, W., De, S., Qin, J., Frank-Kamenetsky, M., Jayaprakash, K. N., Jayaraman, M., Rajeev, K. G., Cantley, W. L., Dorkin, J. R., et al. (2010). Targeted delivery of RNAi therapeutics with endogenous and exogenous ligand-based mechanisms. Molecular Therapy, 18(7), $1357-1364$.

Aldawsari, M., Babu, R. J., \& Chougule, M. B. (2015). Progress in topical SiRNA delivery approaches for skin disorders. Current Pharmaceutical Design, 21, 4594-4605.

Almeida, R., \& Allshire, R. C. (2005). RNA silencing and genome regulation. Trends in Cell Biology, 15(5), 251-258.

Amarzguioui, M., \& Prydz, H. (2004). An algorithm for selection of functional SiRNA sequences. Biochemical and Biophysical Research Communications, 316(4), 1050-1058.

Bae, S., Kim, H., Lee, Y., Xu, X., Park, J. S., Zheng, Y., Balakrishnan, J., Lei, T., Ri Kim, H., Song, Y., II, et al. (2010). Roll-to-roll production of 30-inch graphene films for transparent electrodes. Nature Nanotechnology, 5(8), 574-578. 
Bartelmess, J., Quinn, S. J., \& Giordani, S. (2015). Carbon nanomaterials: Multi-functional agents for biomedical fluorescence and Raman imaging. Chemical Society Reviews, 44(14), 4672-4698.

Benjaminsen, R. V., Mattebjerg, M. A., Henriksen, J. R., Moghimi, S. M., \& Andresen, T. L. (2013). The possible "proton Sponge" effect of polyethylenimine (PEI) does not include change in lysosomal PH. Molecular Therapy, 21(1), 149-157.

Berezikov, E. (2011). Evolution of microRNA diversity and regulation in animals. Nature Reviews. Genetics, 12(12), 846-860.

Bertrand, J. R., Pottier, M., Vekris, A., Opolon, P., Maksimenko, A., \& Malvy, C. (2002). Comparison of antisense oligonucleotides and SiRNAs in cell culture and in vivo. Biochemical and Biophysical Research Communications, 296(4), 1000-1004.

Birmingham, A., Anderson, E. M., Reynolds, A., Ilsley-Tyree, D., Leake, D., Fedorov, Y., Baskerville, S., Maksimova, E., Robinson, K., Karpilow, J., et al. (2006). 3' UTR seed matches, but not overall identity, are associated with RNAi off-targets. Nature Methods, 3(3), 199-204.

Boado, R. J. (2005). RNA interference and nonviral targeted gene therapy of experimental brain cancer. NeuroRx, 2(1), 139-150.

Bodor, N., \& Buchwald, P. (2005). Ophthalmic drug design based on the metabolic activity of the eye: Soft drugs and chemical delivery systems. The AAPS Journal, 7(4), E820-E833.

Bonnet, M. E., Erbacher, P., \& Bolcato-Bellemin, A. L. (2008). Systemic delivery of DNA or SiRNA mediated by linear polyethylenimine (L-PEI) does not induce an inflammatory response. Pharmaceutical Research, 25(12), 2972-2982.

Campochiaro, P. A. (2006). Potential applications for RNAi to probe pathogenesis and develop new treatments for ocular disorders. Gene Therapy, 13(6), 559-562.

Cao, N., Cheng, D., Zou, S., Ai, H., Gao, J., \& Shuai, X. (2011). The synergistic effect of hierarchical assemblies of SiRNA and chemotherapeutic drugs co-delivered into hepatic cancer cells. Biomaterials, 32(8), 2222-2232.

Carthew, R. W., \& Sontheimer, E. J. (2009). Origins and mechanisms of MiRNAs and SiRNAs. Cell, 136(4), 642-655.

Cazzin, C., \& Ring, C. J. A. (2010). Recent advances in the manipulation of murine gene expression and its utility for the study of human neurological disease. Biochimica et Biophysica Acta, Molecular Basis of Disease, 1802(10), 796-807.

Chalk, A. M., Wahlestedt, C., \& Sonnhammer, E. L. L. (2004). Improved and automated prediction of effective SiRNA. Biochemical and Biophysical Research Communications, 319(1), 264-274.

Chang, H., Zhang, Y., Li, L., \& Cheng, Y. (2015). Efficient delivery of small interfering RNA into cancer cells using dodecylated dendrimers. Journal of Materials Chemistry B, 3(41), $8197-8202$.

Chen, A. M., Zhang, M., Wei, D., Stueber, D., Taratula, O., Minko, T., \& He, H. (2009). Co-delivery of doxorubicin and Bcl-2 SiRNA by mesoporous silica nanoparticles enhances the efficacy of chemotherapy in multidrug-resistant cancer cells. Small, 5(23), 2673-2677.

Chiu, Y. L., \& Rana, T. M. (2003). SiRNA function in RNAi: A chemical modification analysis. RNA, 9(9), 1034-1048.

Chougule, M. B. (2012). Current scene and prospective potentials of SiRNA in cancer therapy. Journal of Pharmacogenomics Pharmacoproteomics, 03(05), 3-5.

Clark, S. J. (2007). Action at a distance: Epigenetic silencing of large chromosomal regions in carcinogenesis. Human Molecular Genetics, 16(R1), R88-R95.

Conley, S. M., \& Naash, M. I. (2010). Nanoparticles for retinal gene therapy. Progress in Retinal and Eye Research, 29(5), 376-397.

Cun, D., Krohn, D., Jonas, M., Bunker, M., Whiteside, P., Scurr, D., Foged, C., \& Mørck, H. (2011). European journal of pharmaceutics and biopharmaceutics high loading efficiency and sustained release of SiRNA encapsulated in PLGA nanoparticles: Quality by design optimization and characterization. European Journal of Pharmaceutics and Biopharmaceutics, 77(1), 26-35. 
Czauderna, F. (2003). Structural variations and stabilizing modifications of synthetic SiRNAs in mammalian cells. Nucleic Acids Research, 31(11), 2705-2716.

Czech, M. P., Aouadi, M., \& Tesz, G. J. (2011). RNAi-based therapeutic strategies for metabolic disease 3121. Nature Reviews. Endocrinology, 7, 1759-5037. (Electronic)), 473-484.

Dana, H., Chalbatani, G. M., Mahmoodzadeh, H., Karimloo, R., Rezaiean, O., Moradzadeh, A., Mehmandoost, N., Moazzen, F., Mazraeh, A., Marmari, V., et al. (2017). Molecular mechanisms and biological functions of SiRNA. International Journal of Biomedical Sciences, 13(2), 48-57.

Davis, M. E., Zuckerman, J. E., Choi, C. H. J., Seligson, D., Tolcher, A., Alabi, C. A., Yen, Y., Heidel, J. D., \& Ribas, A. (2010). Evidence of RNAi in humans from systemically administered SiRNA via targeted nanoparticles. Nature, 464(7291), 1067-1070.

de la Fuente, M., Raviña, M., Paolicelli, P., Sanchez, A., Seijo, B., \& Alonso, M. J. (2010). Chitosan-based nanostructures: A delivery platform for ocular therapeutics. Advanced Drug Delivery Reviews, 62(1), 100-117.

Deng, H., Jankovic, J., Guo, Y., Xie, W., \& Le, W. (2005). Small interfering RNA targeting the PINK1 induces apoptosis in dopaminergic cells SH-SY5Y. Biochemical and Biophysical Research Communications, 337(4), 1133-1138.

des Rieux, A., Fievez, V., Garinot, M., Schneider, Y.-J. J., \& Préat, V. (2006). Nanoparticles as potential oral delivery systems of proteins and vaccines: A mechanistic approach. Journal of Controlled Release, 116(1), 1-27.

Dorn, G., Patel, S., Wotherspoon, G., Hemmings-Mieszczak, M., Barclay, J., Natt, F. J. C., Martin, P., Bevan, S., Fox, A., Ganju, P., et al. (2004). SiRNA relieves chronic neuropathic pain. Nucleic Acids Research, 32(5), e49-e49.

Draz, M. S., Fang, B. A., Zhang, P., Hu, Z., Gu, S., Weng, K. C., Gray, J. W., \& Chen, F. F. (2014). Nanoparticle-mediated systemic delivery of SiRNA for treatment of cancers and viral infections. Theranostics, 4(9), 872-892.

Drummond, D. C., Noble, C. O., Guo, Z., Hayes, M. E., Connolly-Ingram, C., Gabriel, B. S., Hann, B., Liu, B., Park, J. W., Hong, K., et al. (2010). Development of a highly stable and targetable nanoliposomal formulation of topotecan. Journal of Controlled Release, 141(1), 13-21.

Duan, L., Yan, Y., Liu, J., Wang, B., Li, P., Hu, Q., \& Chen, W. (2016). Target delivery of small interfering RNAs with vitamin E-coupled nanoparticles for treating hepatitis C. Scientific Reports, 6, 24867.

Durcan, N., Murphy, C., \& Cryan, S. A. (2008). Inhalable SiRNA: Potential as a therapeutic agent in the lungs. Molecular Pharmaceutics, 5(4), 559-566.

Euliss, L. E., DuPont, J. A., Gratton, S., \& DeSimone, J. (2006). Imparting size, shape, and composition control of materials for nanomedicine. Chemical Society Reviews, 35(11), 1095.

Fire, A., Xu, S., Montgomery, M. K., Kostas, S. A., Driver, S. E., \& Mello, C. C. (1998). Potent and specific genetic interference by double-stranded RNA in Caenorhabditis Elegans. Nature, 391(6669), 806-811.

Gavrilov, K., \& Saltzman, W. M. (2012). Therapeutic SiRNA: Principles, challenges, and strategies. The Yale Journal of Biology and Medicine, 85(2), 187-200.

Gewirtz, A. M. (2007). On future's doorstep: RNA interference and the pharmacopeia of tomorrow. The Journal of Clinical Investigation, 117(12), 3612-3614.

Gherardini, L., Bardi, G., Gennaro, M., \& Pizzorusso, T. (2014). Novel SiRNA delivery strategy: A new "Strand" in CNS translational medicine? Cellular and Molecular Life Sciences, 71, 1-20.

Giljohann, D. A., Seferos, D. S., Prigodich, A. E., Patel, P. C., \& Mirkin, C. A. (2009). Gene regulation with polyvalent SiRNA-nanoparticle conjugates. Journal of the American Chemical Society, 131(6), 2072-2073.

Gottesman, M. M. (2002). Mechanisms of cancer drug resistance. Annual Review of Medicine, $53(1), 615-627$.

Griesenbach, U., Kitson, C., Garcia, S. E., Farley, R., Singh, C., Somerton, L., Painter, H., Smith, R. L., Gill, D. R., Hyde, S. C., et al. (2006). Inefficient cationic lipid-mediated SiRNA and antisense oligonucleotide transfer to airway epithelial cells in vivo. Respiratory Research, 7(1), 26. 
Gu, J., Al-Bayati, K., \& Ho, E. A. (2017). Development of antibody-modified chitosan nanoparticles for the targeted delivery of SiRNA across the blood-brain barrier as a strategy for inhibiting HIV replication in astrocytes. Drug Delivery and Translational Research, 7(4), 497-506.

Guo, P., Coban, O., Snead, N. M., Trebley, J., Hoeprich, S., Guo, S., \& Shu, Y. (2010a). Engineering Rna for targeted Sirna delivery and medical application. Advanced Drug Delivery Reviews, 62(6), 650-666.

Guo, S., Huang, Y., Jiang, Q., Sun, Y., Deng, L., Liang, Z., Du, Q., Xing, J., Zhao, Y., Wang, P. C., et al. (2010b). Enhanced gene delivery and SiRNA silencing by gold nanoparticles coated with charge-reversal polyelectrolyte. ACS Nano, 4(9), 5505-5511.

Gutbier, B., Kube, S. M., Reppe, K., Santel, A., Lange, C., Kaufmann, J., Suttorp, N., \& Witzenrath, M. (2010). RNAi-mediated suppression of constitutive pulmonary gene expression by small interfering RNA in mice. Pulmonary Pharmacology \& Therapeutics, 23(4), 334-344.

Hammond, S. M., Bernstein, E., Beach, D., \& Hannon, G. J. (2000). An RNA-directed nuclease mediates post-transcriptional gene silencing in drosophila cells. Nature, 404, 293.

Han, H. D., Mangala, L. S., Lee, J. W., Shahzad, M. M. K., Kim, H. S., Shen, D., Nam, E. J., Mora, E. M., Stone, R. L., Lu, C., et al. (2010). Targeted gene silencing using RGD-labeled chitosan nanoparticles. Clinical Cancer Research, 16(15), 3910-3922.

Hansen, K. M., Ji, H. F., Wu, G., Datar, R., Cote, R., Majumdar, A., \& Thundat, T. (2001). Cantilever-based optical deflection assay for discrimination of DNA single-nucleotide mismatches. Analytical Chemistry, 73(7), 1567-1571.

Harper, S. Q., Staber, P. D., He, X., Eliason, S. L., Martins, I. H., Mao, Q., Yang, L., Kotin, R. M., Paulson, H. L., \& Davidson, B. L. (2005). RNA interference improves motor and neuropathological abnormalities in a Huntington's disease mouse model. Proceedings of the National Academy of Sciences, 102(16), 5820-5825.

Hatakeyama, H., Akita, H., Ito, E., Hayashi, Y., \& Oishi, M. (2011). Biomaterials systemic delivery of SiRNA to tumors using a lipid nanoparticle containing a tumor-Specific cleavable PEGlipid. Biomaterials, 32(18), 4306-4316.

Heale, B. S. E., Soifer, H. S., Bowers, C., \& Rossi, J. J. (2005). SiRNA target site secondary structure predictions using local stable substructures. Nucleic Acids Research, 33(3), 1-10.

Huang, Y., Cheng, Q., Ji, J.-L., Zheng, S., Du, L., Meng, L., Wu, Y., Zhao, D., Wang, X., Lai, L., et al. (2016). Pharmacokinetic behaviors of intravenously administered SiRNA in glandular tissues. Theranostics, 6(10), 1528-1541.

Illum, L., \& Davis, S. S. (1984). The organ uptake of intravenously administered colloidal particles can be altered using a non-ionic surfactant (Poloxamer 338). FEBS Letters, 167(1), 79-82.

Inoue, M., Matsumoto, S., Saito, H., Tsujitani, S., \& Ikeguchi, M. (2008). Intraperitoneal administration of a small interfering RNA targeting nuclear factor-kappa B with paclitaxel successfully prolongs the survival of xenograft model mice with peritoneal metastasis of gastric cancer. International Journal of Cancer, 123(11), 2696-2701.

Jagannath, A., \& Wood, M. (2007). RNA interference based gene therapy for neurological disease. Briefings in Functional Genomics \& Proteomics, 6(1), 40-49.

Jang, S. H., Wientjes, M. G., Lu, D., \& Au, J. L. S. (2003). Drug delivery and transport to solid tumors. Pharmaceutical Research, 20(9), 1337-1350.

Jiang, S., Eltoukhy, A. A., Love, K. T., Langer, R., \& Anderson, D. G. (2013). Lipidoid-coated Iron oxide nanoparticles for efficient DNA and SiRNA delivery. Nano Letters, 13(3), 1059-1064.

Joo, J., Kwon, E. J., Kang, J., Skalak, M., Anglin, E. J., Mann, A. P., Ruoslahti, E., Bhatia, S. N., \& Sailor, M. J. (2016). Porous silicon-graphene oxide core-shell nanoparticles for targeted delivery of SiRNA to the injured brain. Nanoscale Horizons, 1(5), 407-414.

Jung, C. R., Yoo, J., Ye, J. J., Kim, S., Chu, I. S., Young, I. Y., Jong, Y. C., \& Im, D. S. (2006). Adenovirus-mediated transfer of SiRNA against PTTG1 inhibits liver cancer cell growth in vitro and in vivo. Hepatology, 43(5), 1042-1052.

Khatri, N. I., Rathi, M. N., Kolte, A. A., Kore, G. G., Lalan, M. S., Trehan, S., \& Misra, A. R. (2012). Patents review in SiRNA delivery for pulmonary disorders. Recent Patents on Drug Delivery \& Formulation, 6, 45-65. 
Kim, N., Jiang, D., Jacobi, A. M., Lennox, K. A., Rose, S. D., Behlke, M. A., \& Salem, A. K. (2012). Synthesis and characterization of mannosylated pegylated polyethylenimine as a carrier for SiRNA. International Journal of Pharmaceutics, 427(1), 123-133.

Kulhari, H., Pooja, D., Singh, M. K., \& Chauhan, A. S. (2015). Optimization of carboxylateterminated poly(amidoamine) dendrimer-mediated cisplatin formulation. Drug Development and Industrial Pharmacy, 41(2), 232-238.

Cavallaro, G., Sardo, C., Scialabba, C., Licciardi, M., \& Giammona, G. (2017). Smart inulin-based polycationic nanodevices for SiRNA delivery. Current Drug Delivery, 14(2), 224-230.

Landen, C. N., Chavez-Reyes, A., Bucana, C., Schmandt, R., Deavers, M. T., Lopez-Berestein, G., \& Sood, A. K. (2005). Therapeutic EphA2 gene targeting in vivo using neutral liposomal small interfering RNA delivery. Cancer Research, 65(15), 6910 LP-6916918.

Lee, S. H., Bae, K. H., Kim, S. H., Lee, K. R., \& Park, T. G. (2008). Amine-functionalized gold nanoparticles as non-cytotoxic and efficient intracellular SiRNA delivery carriers. International Journal of Pharmaceutics, 364(1), 94-101.

Lee, S. J., Kim, M. J., Kwon, I. C., \& Roberts, T. M. (2016). Delivery strategies and potential targets for SiRNA in major cancer types. Advanced Drug Delivery Reviews, 104, 2-15.

Li, M., Zhang, W., Wang, B., Gao, Y., Song, Z., \& Zheng, Q. C. (2016). Ligand-based targeted therapy: A novel strategy for hepatocellular carcinoma. International Journal of Nanomedicine, $11,5645-5669$.

Li, X., Sun, A. N., Liu, Y. J., Zhang, W. J., Pang, N., Cheng, S. X., \& Qi, X. R. (2018). Amphiphilic dendrimer engineered nanocarrier systems for co-delivery of SiRNA and paclitaxel to matrix metalloproteinase-rich tumors for synergistic therapy. NPG Asia Materials, 10(4), 238-254.

Lian, S., Kang, Z., Wang, E., Jiang, M., Hu, C., \& Xu, L. (2003). Convenient synthesis of single crystalline magnetic Fe3O4 nanorods. Solid State Communications, 127(9-10), 605-608.

Licciardi, M., Tang, Y., Billingham, N. C., Armes, S. P., \& Lewis, A. L. (2005). Synthesis of novel folic acid-functionalized biocompatible block copolymers by atom transfer radical polymerization for gene delivery and encapsulation of hydrophobic drugs. Biomacromolecules, 6(2), $1085-1096$.

Lin, P. J. C., Tam, Y. Y. C., Hafez, I., Sandhu, A., Chen, S., Ciufolini, M. A., Nabi, I. R., \& Cullis, P. R. (2013). Influence of cationic lipid composition on uptake and intracellular processing of lipid nanoparticle formulations of SiRNA. Nanomedicine: Nanotechnology, Biology and Medicine, 9(2), 233-246.

Liu, J., Carmell, M. A., Rivas, F. V., Marsden, C. G., Thomson, J. M., Song, J. J., Hammond, S. M., Joshua-Tor, L., \& Hannon, G. J. (2004). Argonaute2 is the catalytic engine of mammalian RNAi. Science (80-), 305(5689), 1437-1441.

Liu, X., Liu, C., Laurini, E., Posocco, P., Pricl, S., Qu, F., Rocchi, P., \& Peng, L. (2012). Efficient delivery of sticky SiRNA and potent gene silencing in a prostate cancer model using a generation 5 triethanolamine-core PAMAM dendrimer. Molecular Pharmaceutics, 9(3), 470-481.

Lu, J. J., Langer, R., Chen, J. A., \& Novel Mechanism, I. (2009). Involved in cationic lipid-mediated functional SiRNA delivery. Molecular Pharmaceutics, 6(3), 763-771.

Ma, J.-B. B., Yuan, Y.-R. R., Meister, G., Pei, Y., Tuschl, T., \& Patel, D. J. (2005). Structural basis for $5^{\prime}$-end-specific recognition of guide RNA by the A. Fulgidus piwi protein. Nature, 434(7033), 666-670.

Madison, K. C. (2003). Barrier function of the skin: "La raison d'Être" of the epidermis. The Journal of Investigative Dermatology, 121(2), 231-241.

Maeda, H. (2001). The enhanced permeability and retention (EPR) effect in tumor vasculature: The key role of tumor-selective macromolecular drug targeting. Advances in Enzyme Regulation, 41(00), 189-207.

Malek, A., Czubayko, F., \& Aigner, A. P. E. G. (2008). Grafting of polyethylenimine (PEI) exerts different effects on DNA transfection and SiRNA-induced gene targeting efficacy. Journal of Drug Targeting, 16(2), 124-139. 
Malhotra, M., Tomaro-Duchesneau, C., Saha, S., \& Prakash, S. (2013). Systemic SiRNA delivery via peptide-tagged polymeric nanoparticles, targeting PLK1 gene in a mouse xenograft model of colorectal cancer. International Journal of Biomaterials, 2013, 1-13.

Marques, J. T., \& Williams, B. R. G. (2005). Activation of the mammalian immune system by SiRNAs. Nature Biotechnology, 23(11), 1399-1405.

Martinez, J., Patkaniowska, A., Urlaub, H., Lührmann, R., \& Tuschl, T. (2002). Single-stranded antisense SiRNAs guide target RNA cleavage in RNAi. Cell, 110(5), 563-574.

Meister, G., Landthaler, M., Patkaniowska, A., Dorsett, Y., Teng, G., \& Tuschl, T. (2004). Human Argonaute2 mediates RNA cleavage targeted by MiRNAs and SiRNAs. Molecular Cell, 15(2), 185-197.

Mekaru, H., Lu, J., \& Tamanoi, F. (2015). Development of mesoporous silica-based nanoparticles with controlled release capability for cancer therapy. Advanced Drug Delivery Reviews, 95, 40-49.

Meng, H., Liong, M., Xia, T., Li, Z., Ji, Z., Zink, J. I., \& Nel, A. E. (2010). Engineered design of mesoporous silica nanoparticles to deliver doxorubicin and P-glycoprotein SiRNA to overcome drug resistance in a cancer cell line. ACS Nano, 4(8), 4539-4550.

Merkel, O. M., Beyerle, A., Librizzi, D., Pfestroff, A., Behr, T. M., Sproat, B., Barth, P. J., \& Kissel, T. (2009). Nonviral SiRNA delivery to the lung: Investigation of PEG-PEI polyplexes and their in vivo performance. Molecular Pharmaceutics, 6(4), 1246-1260.

Midoux, P., Pichon, C., Yaouanc, J. J., \& Jaffrès, P. A. (2009). Chemical vectors for gene delivery: A current review on polymers, peptides and lipids containing histidine or imidazole as nucleic acids carriers. British Journal of Pharmacology, 157(2), 166-178.

Minakuchi, Y., Takeshita, F., Kosaka, N., Sasaki, H., Yamamoto, Y., Kouno, M., Honma, K., Nagahara, S., Hanai, K., Sano, A., et al. (2004). Atelocollagen-mediated synthetic small interfering RNA delivery for effective gene silencing in vitro and in vivo. Nucleic Acids Research, 32(13), e109-e109.

Minami, K., Okamoto, K., Doi, K., Harano, K., Noiri, E., \& Nakamura, E. (2014). SiRNA delivery targeting to the lung via agglutination-induced accumulation and clearance of cationic tetraamino fullerene. Scientific Reports, 4, 4916.

Mohammadi, M., Salmasi, Z., Hashemi, M., Mosaffa, F., Abnous, K., \& Ramezani, M. (2015). Single-walled carbon nanotubes functionalized with aptamer and piperazine-polyethylenimine derivative for targeted SiRNA delivery into breast cancer cells. International Journal of Pharmaceutics, 485(1-2), 50-60.

Mouldy, S. (2006). Single-stranded small interfering RNA are more immunostimulatory than their double-stranded counterparts: A central role for 2'-hydroxyl uridines in immune responses. European Journal of Immunology, 36(5), 1222-1230.

Naito, Y., \& Ui-Tei, K. (2013). Designing functional SiRNA with reduced off-target effects. Methods in Molecular Biology, 942, 57-68.

Ngamcherdtrakul, W., Morry, J., Gu, S., Castro, D. J., Goodyear, S. M., Sangvanich, T., Reda, M. M., Lee, R., Mihelic, S. A., Beckman, B. L., et al. (2015). Cationic polymer modified mesoporous silica nanoparticles for targeted SiRNA delivery to HER2 + breast cancer. Advanced Functional Materials, 25(18), 2646-2659.

Nguyen, Q. D., Schachar, R. A., Nduaka, C. I., Sperling, M., Basile, A. S., Klamerus, K. J., ChiBurris, K., Yan, E., Paggiarino, D. A., Rosenblatt, I., et al. (2012a). Dose-ranging evaluation of intravitreal SiRNA PF-04523655 for diabetic macular edema (the DEGAS study). Investigative Ophthalmology and Visual Science, 53(12), 7666-7674.

Nguyen, Q. D., Schachar, R. A., Nduaka, C. I., Sperling, M., Basile, A. S., Klamerus, K. J., ChiBurris, K., Yan, E., Paggiarino, D. A., Rosenblatt, I., et al. (2012b). Phase 1 dose-escalation study of a SiRNA targeting the RTP801 gene in age-related macular degeneration patients. Eye, 26(8), 1099-1105.

Nimesh, S., \& Chandra, R. (2009). Polyethylenimine nanoparticles as an efficient in vitro SiRNA delivery system. European Journal of Pharmaceutics and Biopharmaceutics, 73(1), 43-49. 
Nishina, K., Unno, T., Uno, Y., Kubodera, T., Kanouchi, T., Mizusawa, H., \& Yokota, T. (2008). Efficient in vivo delivery of SiRNA to the liver by conjugation of $\alpha$-tocopherol. Molecular Therapy, 16(4), 734-740.

Noble, C. O., Guo, Z., Hayes, M. E., Marks, J. D., Park, J. W., Benz, C. C., Kirpotin, D. B., \& Drummond, D. C. (2009). Characterization of highly stable liposomal and immunoliposomal formulations of vincristine and vinblastine. Cancer Chemotherapy and Pharmacology, 64(4), $741-751$.

Norman, M. E., Williams, P., \& Illum, L. (1993). Influence of block copolymers on the adsorption of plasma proteins to microspheres. Biomaterials, 14(3), 193-202.

Pañeda, C. (2013). SYL040012, a SiRNA for the treatment of glaucoma. Acta Ophthalmologica, 91(s252), 0-0.

Patil, Y. P., \& Jadhav, S. (2014). Novel methods for liposome preparation. Chemistry and Physics of Lipids, 177, 8-18.

Patzel, V., Rutz, S., Dietrich, I., Köberle, C., Scheffold, A., \& Kaufmann, S. H. E. (2005). Design of SiRNAs producing unstructured guide-RNAs results in improved RNA interference efficiency. Nature Biotechnology, 23(11), 1440-1444.

Pecot, C. V., Calin, G. A., Coleman, R. L., Lopez-Berestein, G., \& Sood, A. K. (2011). RNA interference in the clinic: Challenges and future directions. Nature Reviews Cancer, 11(1), 59-67.

Pellish, R. S., Nasir, A., Ramratnam, B., \& Moss, S. F. (2008). Review article: RNA interference - potential therapeutic applications for the gastroenterologist. Alimentary Pharmacology \& Therapeutics, 27(9), 715-723.

Pittella, F., Zhang, M., Lee, Y., Kim, H. J., Tockary, T., Osada, K., Ishii, T., Miyata, K., Nishiyama, N., \& Kataoka, K. (2011). Enhanced endosomal escape of SiRNA-incorporating hybrid nanoparticles from calcium phosphate and PEG-block charge-conversional polymer for efficient gene knockdown with negligible cytotoxicity. Biomaterials, 32(11), 3106-3114.

Pooja, D., Kulhari, H., Singh, M. K., Mukherjee, S., Rachamalla, S. S., \& Sistla, R. (2014). Dendrimer-TPGS mixed micelles for enhanced solubility and cellular toxicity of taxanes. Colloids and Surfaces. B, Biointerfaces, 121, 461-468.

Prausnitz, M. R., Mitragotri, S., \& Langer, R. (2004). Current status and future potential of transdermal drug delivery. Nature Reviews Drug Discovery, 3, 115-124.

Qian, J., \& Berkland, C. (2015). PH-sensitive triblock copolymers for efficient SiRNA encapsulation and delivery. Polymer Chemistry, 6(18), 3472-3479.

Qiu, Y., Lam, J. K. W., Leung, S. W. S., \& Liang, W. (2016a). Delivery of RNAi therapeutics to the airways - From bench to bedside. Molecules, 21, E1249.

Qiu, C., Wei, W., Sun, J., Zhang, H.-T., Ding, J.-S., Wang, J.-C., \& Zhang, Q. (2016b). Systemic delivery of SiRNA by hyaluronan-functionalized calcium phosphate nanoparticles for tumortargeted therapy. Nanoscale, 8(26), 13033-13044.

Ralph, G. S., Radcliffe, P. A., Day, D. M., Carthy, J. M., Leroux, M. A., Lee, D. C. P., Wong, L.-F., Bilsland, L. G., Greensmith, L., Kingsman, S. M., et al. (2005). Silencing mutant SOD1 using RNAi protects against neurodegeneration and extends survival in an ALS model. Nature Medicine, 11, 429.

Rappaport, J., Hanss, B., Kopp, J. B., Copeland, T. D., Bruggeman, L. A., Coffman, T. M., \& Klotman, P. E. (1995). Transport of phosphorothioate oligonucleotides in kidney: Implications for molecular therapy. Kidney International, 47(5), 1462-1469.

Ren, L., Zhang, Y., Cui, C., Bi, Y., \& Ge, X. (2017). Functionalized graphene oxide for antiVEGF SiRNA delivery: Preparation, characterization and evaluation in vitro and in vivo. RSC Advances, 7(33), 20553-20566.

Rettig, G. R., \& Behlke, M. A. (2012). Progress toward in vivo use of SiRNAs-II. Molecular Therapy, 20(3), 483-512.

Rose, S. D., Kim, D. H., Amarzguioui, M., Heidel, J. D., Collingwood, M. A., Davis, M. E., Rossi, J. J., \& Behlke, M. A. (2005). Functional polarity is introduced by dicer processing of short substrate RNAs. Nucleic Acids Research, 33(13), 4140-4156. 
Rossi, J. J. (2009). Cholesterol paves the way for topically applied viricides. Cell Host \& Microbe, $5(1), 6-7$.

Sabbioni, S., Callegari, E., Manservigi, M., Argnani, R., Corallini, A., Negrini, M., \& Manservigi, R. (2006). Use of herpes simplex virus type 1-based amplicon vector for delivery of small interfering RNA. Gene Therapy, 14(5), 459-464.

Sailor, M. J., \& Ji-Ho, P. (2012). Hybrid nanoparticles for detection and treatment of cancer. Advanced Materials, 24(28), 3779-3802.

Santel, A., Aleku, M., Keil, O., Endruschat, J., Esche, V., Fisch, G., Dames, S., Löffler, K., Fechtner, M., Arnold, W., et al. (2006). A novel SiRNA-lipoplex technology for RNA interference in the mouse vascular endothelium. Gene Therapy, 13(16), 1222-1234.

Sawant, R. R., \& Torchilin, V. P. (2012). Multifunctional nanocarriers and intracellular drug delivery. Current Opinion in Solid State \& Materials Science, 16(6), 269-275.

Schedin, F., Geim, A. K., Morozov, S. V., Hill, E. W., Blake, P., Katsnelson, M. I., \& Novoselov, K. S. (2007). Detection of individual gas molecules adsorbed on graphene. Nature Materials, 6(9), 652-655.

Schwarz, D. S., Hutvágner, G., Du, T., Xu, Z., Aronin, N., \& Zamore, P. D. (2003). Asymmetry in the assembly of the RNAi enzyme complex. Cell, 115(2), 199-208.

Sinden, R. R., Pearson, C. E., Potaman, V. N., Ussery, D. W. (1998). DNA: Structure and function. In Verma, R. S. (Ed.). Advances in genome biology (Vol. 5, pp. 1-141).

Singer, O., Marr, R. A., Rockenstein, E., Crews, L., Coufal, N. G., Gage, F. H., Verma, I. M., \& Masliah, E. (2005). Targeting BACE1 with SiRNAs ameliorates Alzheimer disease neuropathology in a transgenic model. Nature Neuroscience, 8(10), 1343-1349.

Singhania, A., Y Wu, S., \& McMillan, N. (2011). Effective delivery of PEGylated SiRNAcontaining lipoplexes to extraperitoneal tumours following intraperitoneal administration. Journal of Drug Delivery, 2011, 192562.

Smith, S. B., Cui, Y., \& Bustamante, C. (1996). Overstretching B-DNA: The elastic response of individual double-stranded and single-stranded DNA molecules. Science (80), 271(5250), 795-799.

Song, J.-J., Liu, J., Tolia, N. H., Schneiderman, J., Smith, S. K., Martienssen, R. A., Hannon, G. J., \& Joshua-Tor, L. (2003). The crystal structure of the Argonaute2 PAZ domain reveals an RNA binding motif in RNAi effector complexes. Nature Structural Biology, 10, 1026.

Song, J.-J., Smith, S., Hannon, G. J., Joshua-Tor, L., Song, J. J., Smith, S. K., Hannon, G. J., \& Joshua-Tor, L. (2004). Crystal structure of Argonaute and its implications for RISC slicer activity. Science, 305, 1434-1437. Vol. 305.

Song, E., Zhu, P., Lee, S.-K., Chowdhury, D., Kussman, S., Dykxhoorn, D. M., Feng, Y., Palliser, D., Weiner, D. B., Shankar, P., et al. (2005). Antibody mediated in vivo delivery of small interfering RNAs via cell-surface receptors. Nature Biotechnology, 23(6), 709-717.

Svenson, S., \& Tomalia, D. A. (2012). Dendrimers in biomedical applications-reflections on the field. Advanced Drug Delivery Reviews, 64(SUPPL), 102-115.

Tang, G. (2005). SiRNA and MiRNA: An insight into RISCs. Trends in Biochemical Sciences, 30(2), 106-114.

Taratula, O., Garbuzenko, O. B., Kirkpatrick, P., Pandya, I., Savla, R., Pozharov, V. P., He, H., \& Minko, T. (2009). Surface-engineered targeted PPI dendrimer for efficient intracellular and intratumoral SiRNA delivery. Journal of Controlled Release, 140(3), 284-293.

Tatiparti, K., Sau, S., Kashaw, S., \& Iyer, A. (2017). SiRNA delivery strategies: A comprehensive review of recent developments. Nanomaterials, 7(4), 77.

Thomas, M., Lu, J. J., Chen, J., \& Klibanov, A. M. (2007). Non-viral SiRNA delivery to the lung. Advanced Drug Delivery Reviews, 59(2-3), 124-133.

Torchilin, V. P. (2014). Multifunctional, stimuli-sensitive nanoparticulate systems for drug delivery. Nature Reviews. Drug Discovery, 13(11), 813-827.

Tseng, Y. C., Mozumdar, S., \& Huang, L. (2009). Lipid-based systemic delivery of SiRNA. Advanced Drug Delivery Reviews, 61(9), 721-731. 
Urbiola, K., Blanco-Fernández, L., Ogris, M., Rödl, W., Wagner, E., \& de Ilarduya, C. T. (2018). Novel PAMAM-PEG-peptide conjugates for SiRNA delivery targeted to the transferrin and epidermal growth factor receptors. Journal of Personalized Medicine, 8(1), 4.

Varkouhi, A. K., Foillard, S., Lammers, T., Schiffelers, R. M., Doris, E., Hennink, W. E., \& Storm, G. (2011). SiRNA delivery with functionalized carbon nanotubes. International Journal of Pharmaceutics, 416(2), 419-425.

Vauthier, C., Zandanel, C., \& Ramon, A. L. (2013). Chitosan-based nanoparticles for in vivo delivery of interfering agents including SiRNA. Current Opinion in Colloid \& Interface Science, 18(5), 406-418.

Verma, P., Prajapati, S. K., \& Prajapati, R. N. (2012). Performance evaluation of Pamam dendrimer based clotrimazole formulations. International Journal of Pharmaceutics, 2. (October), 203-209.

Wang, J., Lu, Z., Wientjes, M. G., \& Au, J. L.-S. (2010). Delivery of SiRNA therapeutics: Barriers and carriers. The AAPS Journal, 12(4), 492-503.

Wang, F., Gao, L., Meng, L. Y., Xie, J. M., Xiong, J. W., \& Luo, Y. A. (2016). Neutralized noncharged polyethylenimine-based system for efficient delivery of SiRNA into heart without toxicity. ACS Applied Materials \& Interfaces, 8(49), 33529-33538.

Wartiovaara, J., Öfverstedt, L. G., Khoshnoodi, J., Zhang, J., Mäkelä, E., Sandin, S., Ruotsalainen, V., Cheng, R. H., Jalanko, H., Skoglund, U., et al. (2004). Nephrin strands contribute to a porous slit diaphragm scaffold as revealed by electron tomography. The Journal of Clinical Investigation, 114(10), 1475-1483.

Watanabe, K., Harada-Shiba, M., Suzuki, A., Gokuden, R., Kurihara, R., Sugao, Y., Mori, T., Katayama, Y., \& Niidome, T. (2009). In vivo SiRNA delivery with dendritic poly(1-lysine) for the treatment of hypercholesterolemia. Molecular BioSystems, 5(11), 1306.

Whitehead, K. A., Langer, R., \& Anderson, D. G. (2009). Knocking down barriers: Advances in SiRNA delivery. Nature Reviews Drug Discovery, 8(2), 129-138.

Wilson, R. C., \& Doudna, J. A. (2013). Molecular mechanisms of RNA interference. Annual Review of Biophysics, 42(1), 217-239.

Wolff, J. A., \& Rozema, D. B. (2008). Breaking the bonds: Non-viral vectors become chemically dynamic. Molecular Therapy, 16(1), 8-15.

Wolfrum, C., Shi, S., Jayaprakash, K. N., Jayaraman, M., Wang, G., Pandey, R. K., Rajeev, K. G., Nakayama, T., Charrise, K., Ndungo, E. M., et al. (2007). Mechanisms and optimization of in vivo delivery of lipophilic SiRNAs. Nature Biotechnology, 25(10), 1149-1157.

Wu, S. Y., Chang, H. I., Burgess, M., \& McMillan, N. A. J. (2011). Vaginal delivery of SiRNA using a novel PEGylated lipoplex-entrapped alginate scaffold system. Journal of Controlled Release, 155(3), 418-426.

Wu, C., Gong, F., Pang, P., Shen, M., Zhu, K., Cheng, D., Liu, Z., \& Shan, H. (2013). An RGDmodified MRI-visible polymeric vector for targeted SiRNA delivery to hepatocellular carcinoma in nude mice. PLoS One, 8(6), e66416.

Wu, J., Qu, W., Williford, J. M., Ren, Y., Jiang, X., Jiang, X., Pan, D., Mao, H. Q., \& Luijten, E. (2017). Improved SiRNA delivery efficiency via solvent-induced condensation of micellar nanoparticles. Nanotechnology, 28(20), 204002.

Xia, Y., Tian, J., \& Chen, X. (2016). Biomaterials effect of surface properties on liposomal SiRNA delivery. Biomaterials, 79, 56-68.

Xia, Y., Lin, Z., Li, Y., Zhao, M., Wang, C., Guo, M., Zhang, B., \& Zhu, B. (2017). Targeted delivery of SiRNA using RGDfC-conjugated functionalized selenium nanoparticles for anticancer therapy. Journal of Materials Chemistry B, 5(33), 6941-6952.

Xu, C. F., \& Wang, J. (2015). Delivery systems for SiRNA drug development in cancer therapy. Asian Journal of Pharmaceutical Sciences, 10(1), 1-12.

Yang, Y., Li, J., Liu, F., \& Huang, L. (2012). Systemic delivery of SiRNA via LCP nanoparticle efficiently inhibits lung metastasis. Molecular Therapy, 20(3), 609-615. 
Yezhelyev, M. V., Qi, L., O'Regan, R. M., Nie, S., \& Gao, X. (2008). Proton-sponge coated quantum dots for SiRNA delivery and intracellular imaging. Journal of the American Chemical Society, 130(28), 9006-9012.

Yin, F., Hu, K., Chen, Y., Yu, M., Wang, D., Wang, Q., Yong, K. T., Lu, F., Liang, Y., \& Li, Z. (2017). SiRNA delivery with PEGylated graphene oxide nanosheets for combined photothermal and genetherapy for pancreatic cancer. Theranostics, 7(5), 1133-1148.

Young, S. W. S., Stenzel, M., \& Jia-Lin, Y. (2016). Nanoparticle-SiRNA: A potential cancer therapy? Critical Reviews in Oncology/Hematology, 98, 159-169.

Yu, H., Zou, Y., Wang, Y., Huang, X., Huang, G., Sumer, B. D., Boothman, D. A., \& Gao, J. (2011). Overcoming endosomal barrier by amphotericin B-loaded dual PH-responsive PDMA- b-PDPA micelleplexes for SiRNA delivery. ACS Nano, 5(11), 9246-9255.

Yu-Wai-Man, C., Tagalakis, A. D., Manunta, M. D., Hart, S. L., \& Khaw, P. T. (2016). Receptortargeted liposome-peptide-SiRNA nanoparticles represent an efficient delivery system for MRTF silencing in conjunctival fibrosis. Scientific Reports, 6, 21881.

Zeng, Y., Yang, Z., Li, H., Hao, Y., Liu, C., Zhu, L., Liu, J., Lu, B., \& Li, R. (2017). Multifunctional nanographene oxide for targeted gene-mediated thermochemotherapy of drug-resistant tumour. Scientific Reports, 7, 43506.

Zhang, Y., Cristofaro, P., Silbermann, R., Pusch, O., Boden, D., Konkin, T., Hovanesian, V., Monfils, P. R., Resnick, M., Moss, S. F., et al. (2006). Engineering mucosal RNA interference in vivo. Molecular Therapy, 14(3), 336-342.

Zhang, Y.-M., Yang, Y., Zhang, Y., \& Liu, Y. (2016). Polysaccharide nanoparticles for efficient SiRNA targeting in cancer cells by supramolecular PKa shift. Scientific Reports, 6(28848), $1-11$.

Zhou, J., Patel, T. R., Fu, M., Bertram, J. P., \& Saltzman, W. M. (2012). Octa-functional PLGA nanoparticles for targeted and efficient SiRNA delivery to tumors. Biomaterials, 33(2), 583-591.

Zhou, J., Wu, Y., Wang, C., Cheng, Q., Han, S., Wang, X., Zhang, J., Deng, L., Zhao, D., Du, L., et al. (2016). PH-sensitive nanomicelles for high-efficiency SiRNA delivery in vitro and in vivo: An insight into the design of polycations with robust cytosolic release. Nano Letters, 16(11), 6916-6923.

Zhu, L., Perche, F., Wang, T., \& Torchilin, V. P. (2014). Matrix metalloproteinase 2-sensitive multifunctional polymeric micelles for tumor-specific co-delivery of SiRNA and hydrophobic drugs. Biomaterials, 35(13), 4213-4222.

Zhu, H., Liu, W., Cheng, Z., Yao, K., Yang, Y., Xu, B., \& Su, G. (2017). Targeted delivery of SiRNA with PH-responsive hybrid gold nanostars for cancer treatment. International Journal of Molecular Sciences, 18(10), 2029. 